\title{
Lorentz-violating extension of the standard model
}

\author{
D. Colladay and V. Alan Kostelecký \\ Physics Department, Indiana University, Bloomington, Indiana 47405
}

(Received 24 June 1998; published 26 October 1998)

\begin{abstract}
In the context of conventional quantum field theory, we present a general Lorentz-violating extension of the minimal $\mathrm{SU}(3) \times \mathrm{SU}(2) \times \mathrm{U}(1)$ standard model including $C P T$-even and $C P T$-odd terms. It can be viewed as the low-energy limit of a physically relevant fundamental theory with Lorentz-covariant dynamics in which spontaneous Lorentz violation occurs. The extension has gauge invariance, energy-momentum conservation, and covariance under observer rotations and boosts, while covariance under particle rotations and boosts is broken. The quantized theory is Hermitian and power-counting renormalizable, and other desirable features such as microcausality, positivity of the energy, and the usual anomaly cancellation are expected. Spontaneous symmetry breaking to the electromagnetic $\mathrm{U}(1)$ is maintained, although the Higgs expectation is shifted by a small amount relative to its usual value and the $Z^{0}$ field acquires a small expectation. A general Lorentzbreaking extension of quantum electrodynamics is extracted from the theory, and some experimental tests are considered. In particular, we study modifications to photon behavior. One possible effect is vacuum birefringence, which could be bounded from cosmological observations by experiments using existing techniques. Radiative corrections to the photon propagator are examined. They are compatible with spontaneous Lorentz and $C P T$ violation in the fermion sector at levels suggested by Planck-scale physics and accessible to other terrestrial laboratory experiments. [S0556-2821(99)01601-X]

PACS number(s): 11.30.Er, 12.60.-i, 12.20.Fv, 41.20.Jb
\end{abstract}

\section{INTRODUCTION}

The minimal $\mathrm{SU}(3) \times \mathrm{SU}(2) \times \mathrm{U}(1)$ standard model, although phenomenologically successful, leaves unresolved a variety of issues. It is believed to be the low-energy limit of a fundamental theory that also provides a quantum description of gravitation. An interesting question is whether any aspects of this underlying theory could be revealed through definite experimental signals accessible with present techniques.

The natural scale for a fundamental theory including gravity is governed by the Planck mass $M_{P}$, which is about 17 orders of magnitude greater than the electroweak scale $m_{W}$ associated with the standard model. This suggests that observable experimental signals from a fundamental theory might be expected to be suppressed by some power of the ratio $r \approx m_{W} / M_{P} \simeq 10^{-17}$. Detection of these minuscule effects at present energy scales would be likely to require experiments of exceptional sensitivity, preferably ones seeking to observe a signal forbidden in conventional renormalizable gauge theories.

To identify signals of this type, one approach is to examine proposed fundamental theories for effects that are qualitatively different from standard-model physics. For example, at present the most promising framework for a fundamental theory is string $(M)$ theory. The qualitative difference between particles and strings means that qualitatively new physics is expected at the Planck scale. An interesting challenge would be to determine whether this could lead to observable low-energy effects.

In the present work, we consider the possibility that the new physics involves a violation of Lorentz symmetry. It has been shown that spontaneous Lorentz breaking may occur in the context of string theories with Lorentz-covariant dynamics [1]. Unlike the conventional standard model, string theo- ries typically involve interactions that could destabilize the naive vacuum and trigger the generation of nonzero expectation values for Lorentz tensors. Note that some kind of spontaneous breaking of the higher-dimensional Lorentz symmetry is expected in any realistic Lorentz-covariant fundamental theory involving more than four spacetime dimensions. If the breaking extends into the four macroscopic spacetime dimensions, apparent Lorentz violation could occur at the level of the standard model. This would represent a possible observable effect from the fundamental theory, originating outside the structure of conventional renormalizable gauge models.

A framework has been developed for treating the effects of spontaneous Lorentz breaking in the context of a lowenergy effective theory [2], where certain terms can be induced that appear to violate Lorentz invariance explicitly. It turns out that, from a theoretical perspective, the resulting effects are comparatively minimal.

An important point is that Lorentz symmetry remains a property of the underlying fundamental theory because the breaking is spontaneous. This implies that various attractive features of conventional theories, including microcausality and positivity of the energy, are expected to hold in the lowenergy effective theory. Also, energy and momentum are conserved as usual, provided the tensor expectation values in the fundamental theory are spacetime-position independent. Moreover, standard quantization methods are unaffected, so a relativistic Dirac equation and a nonrelativistic Schrödinger equation emerge in the appropriate limits.

Another important aspect of the spontaneous breaking is that both the fundamental theory and the effective lowenergy theory remain invariant under observer Lorentz transformations, i.e., rotations or boosts of an observer's inertial frame [2]. The presence of nonzero tensor expectation values in the vacuum affects only invariance properties under par- 
ticle Lorentz transformations, i.e., rotations or boosts of a localized particle or field that leave unchanged the background expectation values.

This framework for treating spontaneous Lorentz violation has been used to obtain a general extension of the minimal $\mathrm{SU}(3) \times \mathrm{SU}(2) \times \mathrm{U}(1)$ standard model that violates both Lorentz invariance and $C P T$ [2]. In addition to the desirable features of energy-momentum conservation, observer Lorentz invariance, conventional quantization, hermiticity, and the expected microcausality and positivity of the energy, this standard-model extension maintains gauge invariance and power-counting renormalizability. It would emerge from any fundamental theory (not necessarily string theory) that generates the standard model and contains spontaneous Lorentz and $C P T$ violation.

The present work continues our previous theoretical investigations of spontaneous Lorentz and $C P T$ breaking. Working first at the level of the standard model, we provide explicitly in Sec. II the full Lorentz-violating extension, including the $C P T$-even Lorentz-breaking terms described implicitly in Ref. [2]. We also give some details of the modifications to the usual electroweak symmetry breaking.

Since many sensitive measures of Lorentz and $C P T$ symmetry involve tests of quantum electrodynamics (QED), it is useful to extract from the standard-model extension a generalized QED that allows for possible Lorentz and $C P T$ violations. This extended QED, given in Sec. III, involves modifications of the usual QED in both the fermion and the photon sectors. Some comments are also given in Sec. III about the implications of this theory for experimental tests with electrons and positrons.

In the remainder of this paper, we focus primarily on the photon sector of the extended QED, presenting a study of the theoretical and experimental implications of the modifications to photon properties arising from the possible Lorentz and $C P T$ violations. Section IV discusses changes in the basic theory, including the modified Maxwell equations and properties of their solutions. One possible effect is vacuum photon birefringence, and some associated features are described. We show that feasible measurements limiting birefringence on cosmological scales could tightly constrain the Lorentz-violating terms. In Sec. V, some important consistency checks on the theory at the level of radiative corrections are presented, largely at the one-loop level. The types of Lorentz violation that can be affected by radiative corrections are identified, and explicit calculations are given. We show that the effects are compatible with spontaneous Lorentz and $C P T$ violation in the fermion sector at levels accessible to other QED experiments.

Since the standard-model extension provides a quantitative microscopic theory of Lorentz and $C P T$ violation, it is feasible to identify potentially observable signals and to establish bounds from various experiments other than ones in the photon sector. Numerous tests of Lorentz invariance and $C P T$ exist. The present theory provides a single coherent framework at the level of the standard model and QED that can be used as a basis for the analysis and comparison of these tests. Although many experiments are insensitive to the suppressed effects motivating our investigation, certain high- precision ones might have observable signals within this framework. In particular, the results in the present paper have been used to examine possible bounds on $C P T$ and Lorentz violations from measurements of neutral-meson oscillations [3-6], from tests of QED in Penning traps [7,8], and from baryogenesis [9]. Several other investigations are underway, including a study [10] of possible Lorentz and $C P T$ effects on hydrogen and antihydrogen spectroscopy [11] and another [12] of limits attainable in clockcomparison experiments [13].

The analyses of the standard-model and QED extensions performed in the present work leave unaddressed a number of significant theoretical issues arising at scales between the electroweak mass and the Planck mass. These include the "dimension problem" of establishing whether spontaneous Lorentz breaking in the fundamental theory near the Planck scale indeed extends to the four physical spacetime dimensions and, if so, the mechanism for its suppression or, if not, why exactly four spacetime dimensions are spared. Other issues include the effects of mode fluctuations around the tensor expectation values and possible constraints and effects arising from a nonminimal standard model or (super)unification below the Planck scale.

Another potentially important topic is the implication of spontaneous Lorentz violation for gravity at observable energies. Like the usual standard model, the standard-model extension considered here disregards gravitational effects. The particle Lorentz symmetry that is broken in this theory is therefore effectively a global symmetry, and so one might expect Nambu-Goldstone modes. Since gravity is associated with local Lorentz invariance, it is natural to ask about the role of these modes in a version of the standard-model extension that includes gravity. In a gauge theory, when a suitable scalar acquires a nonzero expectation value, the Higgs mechanism occurs: the propagator for the gauge boson is modified, and a mass is generated. Similarly, in a theory with gravitational couplings, when a Lorentz tensor acquires a nonzero expectation value, the graviton propagator can be modified. However, no mass for the graviton is induced because the gravitational connection is related to the derivative of the metric rather than to the metric itself [1]. In this sense, there is no gravitational Higgs effect.

The theory described here appears at present to be the sole candidate for a consistent extension of the standard model providing a microscopic theory of Lorentz violation. A complete review of alternative approaches to possible Lorentz and $C P T$ violation lies beyond the scope of this paper. Works known to us of relevance in the present context are referenced in the body of the text below. Among other ideas in the literature are several distinctive ones developed from perspectives very different from ours. Following early work by Dirac and Heisenberg, several authors have considered an unphysical spontaneous Lorentz breaking in an effort to interpret the photon as a Nambu-Goldstone boson [14]. Nielsen and his colleagues have suggested the converse of the philosophy in the present work: that the observed Lorentz symmetry in nature might be a low-energy manifestation of a fundamental theory without Lorentz invariance. A discussion of this idea and a brief review of the literature on Lorentz 
breaking prior to the establishment of the usual minimal standard model may be found in Ref. [15]. Hawking has suggested [16] the possibility that conventional quantum mechanics is invalidated by gravitational effects and that this might lead to $C P T$ violation, among other effects. The implications for experiments in the kaon system [17] are known to be entirely different from those arising in the present standard-model extension, which is based on conventional quantum theory. There is also a body of literature pertaining to unconventional theories of gravity (without standardmodel physics), among which are some models containing various possible sources of local Lorentz violation [18].

\section{STANDARD-MODEL EXTENSION}

In this section, we extend the minimal standard model by adding all possible Lorentz-violating terms that could arise from spontaneous symmetry breaking at a fundamental level but that preserve $\mathrm{SU}(3) \times \mathrm{SU}(2) \times \mathrm{U}(1)$ gauge invariance and power-counting renormalizability. Terms that are odd under $C P T$ are explicitly given in Ref. [2] but are also included here for completeness.

The general form of a Lorentz-violating term involves a part that acts as a coupling coefficient and a part constructed from the basic fields in the standard model. The requirements of the derivation impose various limitations on the possible structures of both parts. Taken together, these requirements place significant constraints on the form of terms in the standard-model extension.

The part acting as a coupling coefficient carries spacetime indices reflecting the properties under observer Lorentz transformations of the relevant nonzero expectation values from the fundamental theory. The coupling coefficient may be complex, but it is constrained by the requirement that the Lagrangian be Hermitian. For a coupling coefficient with an even number of spacetime indices, the pure trace component is irrelevant for present purposes because it maintains Lorentz invariance. A coupling coefficient of this type can therefore be taken traceless.

The field part may involve covariant derivatives and, if fermions are involved, $\gamma$ matrices. Gauge invariance requires that the field part be a singlet under $\mathrm{SU}(3) \times \mathrm{SU}(2) \times \mathrm{U}(1)$, while power-counting renormalizability implies that it must have mass dimension no greater than four. The requirement that the standard-model extension originates from spontaneous Lorentz breaking in a covariant fundamental theory implies the whole Lorentz-violating term must be a singlet under observer Lorentz transformations, so the field part must have indices matching those of the coupling coefficient.

Following the discussion in the introduction, all coupling coefficients are assumed to be heavily suppressed by some power of the ratio $r$ of the light scale to the Planck scale. In the absence of a satisfactory explanation of the suppression mechanism, it would seem premature to attempt specific detailed predictions about the relative sizes of different coupling coefficients. As a possible working hypothesis, one might attribute comparable suppression factors to all terms at the level of the standard-model extension. Note that a term with the field part having mass dimension $n$ must have a coupling coefficient with mass dimension $4-n$, and the relevant scale for these effects is roughly the Planck mass. The hypothesis would therefore suggest that in the low-energy theory a term with the field part of mass dimension $n+1$ would have a coupling coefficient suppressed by an additional power of $r$ relative to the coefficient of a field term of mass dimension $n$. This scheme would be compatible with interpreting the standard model as an effective field theory, in which each additional derivative coupling would involve an additional suppression factor in the coupling coefficient. It would imply a distinct hierarchy among the coupling coefficients introduced below, and would suggest that certain derivative couplings could be neglected relative to comparable nonderivative ones. However, since this hypothesis presently has no basis in a detailed theory, in what follows we have chosen to retain on an equal footing all renormalizable terms compatible with the gauge symmetries of the standard model and with an origin in spontaneous Lorentz breaking.

In what follows, we denote the left- and right-handed lepton and quark multiplets by

$$
\begin{gathered}
L_{A}=\left(\begin{array}{c}
\nu_{A} \\
l_{A}
\end{array}\right)_{L}, \quad R_{A}=\left(l_{A}\right)_{R}, \\
Q_{A}=\left(\begin{array}{c}
u_{A} \\
d_{A}
\end{array}\right)_{L}, \quad U_{A}=\left(u_{A}\right)_{R}, \quad D_{A}=\left(d_{A}\right)_{R},
\end{gathered}
$$

where

$$
\psi_{L} \equiv \frac{1}{2}\left(1-\gamma_{5}\right) \psi, \quad \psi_{R} \equiv \frac{1}{2}\left(1+\gamma_{5}\right) \psi,
$$

as usual, and where $A=1,2,3$ labels the flavor: $l_{A}$ $\equiv(e, \mu, \tau), \quad \nu_{A} \equiv\left(\nu_{e}, \nu_{\mu}, \nu_{\tau}\right), \quad u_{A} \equiv(u, c, t), \quad d_{A} \equiv(d, s, b)$. We denote the Higgs doublet by $\phi$, and in unitary gauge we represent it as

$$
\phi=\frac{1}{\sqrt{2}}\left(\begin{array}{c}
0 \\
r_{\phi}
\end{array}\right)
$$

The conjugate doublet is $\phi^{c}$. The $\mathrm{SU}(3), \mathrm{SU}(2)$, and $\mathrm{U}(1)$ gauge fields are denoted by $G_{\mu}, W_{\mu}$, and $B_{\mu}$, respectively. The corresponding field strengths are $G_{\mu \nu}, W_{\mu \nu}$, and $B_{\mu \nu}$, with the first two understood to be Hermitian adjoint matrices while $B_{\mu \nu}$ is a Hermitian singlet. The corresponding couplings are $g_{3}, g$, and $g^{\prime}$. The electromagnetic U(1) charge $q$ and the angle $\theta_{W}$ are defined through $q=g \sin \theta_{W}$ $=g^{\prime} \cos \theta_{W}$, as usual. The covariant derivative is denoted by $D_{\mu}$, and $A \overleftrightarrow{\partial}_{\mu} B \equiv A \partial_{\mu} B-\left(\partial_{\mu} A\right) B$. The Yukawa couplings are $G_{L}, G_{U}, G_{D}$. Throughout most of this work we use natural units, which could be obtained from the SI system by redefining $\hbar=c=\epsilon_{0}=1$, and we adopt the Minkowski metric $\eta_{\mu \nu}$ with $\eta_{00}=+1$.

The complete Lagrangian for the Lorentz-breaking standard-model extension can be separated into a sum of terms. For completeness, we first provide the Lagrangian terms in the usual $\mathrm{SU}(3) \times \mathrm{SU}(2) \times \mathrm{U}(1)$ minimal standard model:

$$
\mathcal{L}_{\text {lepton }}=\frac{1}{2} i \bar{L}_{A} \gamma^{\mu} \overleftrightarrow{D}_{\mu} L_{A}+\frac{1}{2} i \bar{R}_{A} \gamma^{\mu} \overleftrightarrow{D}_{\mu} R_{A},
$$


$\mathcal{L}_{\text {quark }}=\frac{1}{2} i \bar{Q}_{A} \gamma^{\mu} \overleftrightarrow{D}_{\mu} Q_{A}+\frac{1}{2} i \bar{U}_{A} \gamma^{\mu} \overleftrightarrow{D}_{\mu} U_{A}+\frac{1}{2} i \bar{D}_{A} \gamma^{\mu} \overleftrightarrow{D}_{\mu} D_{A}$

$$
\begin{aligned}
\mathcal{L}_{\text {Yukawa }}= & -\left[\left(G_{L}\right)_{A B} \bar{L}_{A} \phi R_{B}+\left(G_{U}\right)_{A B} \bar{Q}_{A} \phi^{c} U_{B}\right. \\
& \left.+\left(G_{D}\right)_{A B} \bar{Q}_{A} \phi D_{B}\right]+ \text { H.c. },
\end{aligned}
$$

$$
\mathcal{L}_{\mathrm{Higgs}}=\left(D_{\mu} \phi\right)^{\dagger} D^{\mu} \phi+\mu^{2} \phi^{\dagger} \phi-\frac{\lambda}{3 !}\left(\phi^{\dagger} \phi\right)^{2},
$$

$$
\mathcal{L}_{\text {gauge }}=-\frac{1}{2} \operatorname{Tr}\left(G_{\mu \nu} G^{\mu \nu}\right)-\frac{1}{2} \operatorname{Tr}\left(W_{\mu \nu} W^{\mu \nu}\right)-\frac{1}{4} B_{\mu \nu} B^{\mu \nu} .
$$

The usual $\theta$ terms have been omitted, and possible analogous total-derivative terms that break Lorentz symmetry are disregarded in this work.

In the fermion sector of the standard-model extension, the contribution to the Lagrangian can be divided into four parts according to whether the term is $C P T$ even or odd and whether it involves leptons or quarks:

$$
\begin{gathered}
\mathcal{L}_{\text {lepton }}^{\text {CPT-even }}=\frac{1}{2} i\left(c_{L}\right)_{\mu \nu A B} \bar{L}_{A} \gamma^{\mu} \overleftrightarrow{D}^{\nu} L_{B} \\
+\frac{1}{2} i\left(c_{R}\right)_{\mu \nu A B} \bar{R}_{A} \gamma^{\mu} \overleftrightarrow{D}^{\nu} R_{B}, \\
\mathcal{L}_{\text {lepton }}^{\text {CPT-odd }}=-\left(a_{L}\right)_{\mu A B} \bar{L}_{A} \gamma^{\mu} L_{B}-\left(a_{R}\right)_{\mu A B} \bar{R}_{A} \gamma^{\mu} R_{B}, \\
\mathcal{L}_{\text {quark }}^{\text {CPT-even }}=\frac{1}{2} i\left(c_{Q}\right)_{\mu \nu A B} \bar{Q}_{A} \gamma^{\mu} \overleftrightarrow{D}^{\nu} Q_{B} \\
+\frac{1}{2} i\left(c_{U}\right)_{\mu \nu A B} \bar{U}_{A} \gamma^{\mu} \overleftrightarrow{D}^{\nu} U_{B} \\
+\frac{1}{2} i\left(c_{D}\right)_{\mu \nu A B} \bar{D}_{A} \gamma^{\mu} \overleftrightarrow{D}^{\nu} D_{B}, \\
\mathcal{L}_{\text {quark }}^{\text {CPT-odd }}=-\left(a_{Q}\right)_{\mu A B} \bar{Q}_{A} \gamma^{\mu} Q_{B} \\
-\left(a_{U}\right)_{\mu A B} \bar{U}_{A} \gamma^{\mu} U_{B}-\left(a_{D}\right)_{\mu A B} \bar{D}_{A} \gamma^{\mu} D_{B} .
\end{gathered}
$$

In these equations, the various coupling coefficients $c_{\mu \nu}$ and $a_{\mu}$ are understood to be Hermitian in generation space. The coefficients $a_{\mu}$ have dimensions of mass. The dimensionless coefficients $c_{\mu \nu}$ can have both symmetric and antisymmetric parts but can be assumed traceless. A nonzero trace would not contribute to Lorentz violation and in any case can be absorbed by a conventional field normalization ensuring the usual kinetic operator for the matter fields.

The standard-model extension also contains Lorentzviolating couplings between the fermions and the Higgs field, having the gauge structure of the usual Yukawa couplings but involving nontrivial $\gamma$ matrices. These terms are all $C P T$ even:

$$
\begin{aligned}
\mathcal{L}_{\text {Yukawa }}^{\text {CPT-even }}= & -\frac{1}{2}\left[\left(H_{L}\right)_{\mu \nu A B} \bar{L}_{A} \phi \sigma^{\mu \nu} R_{B}\right. \\
& +\left(H_{U}\right)_{\mu \nu A B} \bar{Q}_{A} \phi^{c} \sigma^{\mu \nu} U_{B} \\
& \left.+\left(H_{D}\right)_{\mu \nu A B} \bar{Q}_{A} \phi \sigma^{\mu \nu} D_{B}\right]+ \text { H.c. }
\end{aligned}
$$

The coefficients $H_{\mu \nu}$ are dimensionless and antisymmetric, but like the Yukawa couplings $G_{L, U, D}$ they are not necessarily Hermitian in generation space.

The possible contributions in the Higgs sector can be $C P T$ even or $C P T$ odd:

$$
\begin{aligned}
\mathcal{L}_{\text {Higgs }}^{\text {CPT-even }}= & \frac{1}{2}\left(k_{\phi \phi}\right)^{\mu \nu}\left(D_{\mu} \phi\right)^{\dagger} D_{\nu} \phi+\text { H.c. } \\
& -\frac{1}{2}\left(k_{\phi B}\right)^{\mu \nu} \phi^{\dagger} \phi B_{\mu \nu}-\frac{1}{2}\left(k_{\phi W}\right)^{\mu \nu} \phi^{\dagger} W_{\mu \nu} \phi, \\
& \mathcal{L}_{\text {Higgs }}^{\text {CPT-odd }}=i\left(k_{\phi}\right)^{\mu} \phi^{\dagger} D_{\mu} \phi+\text { H.c. }
\end{aligned}
$$

In Eq. (14), the dimensionless coefficient $k_{\phi \phi}$ can have symmetric real and antisymmetric imaginary parts. The other coefficients in Eq. (14) have dimensions of mass and must be real antisymmetric. The coefficient $k_{\phi}$ for the $C P T$-odd term (15) also has dimensions of mass and can be an arbitrary complex number.

The gauge sector has both $C P T$-even and $C P T$-odd contributions. The $C P T$-even ones are

$$
\begin{aligned}
\mathcal{L}_{\text {gauge }}^{\text {CPT-even }}= & -\frac{1}{2}\left(k_{G}\right)_{\kappa \lambda \mu \nu} \operatorname{Tr}\left(G^{\kappa \lambda} G^{\mu \nu}\right) \\
& -\frac{1}{2}\left(k_{W}\right)_{\kappa \lambda \mu \nu} \operatorname{Tr}\left(W^{\kappa \lambda} W^{\mu \nu}\right) \\
& -\frac{1}{4}\left(k_{B}\right)_{\kappa \lambda \mu \nu} B^{\kappa \lambda} B^{\mu \nu} .
\end{aligned}
$$

In this equation, the dimensionless coefficients $k_{G, W, B}$ are real. They must have the symmetries of the Riemann tensor and a vanishing double trace. The point is that any totally antisymmetric part involves only a total derivative in the Lagrangian density, while a nonzero double trace can be absorbed into a redefinition of the normalization of the corresponding kinetic term (8).

The $C P T$-odd gauge terms are given by the following expression [19]:

$$
\begin{aligned}
\mathcal{L}_{\text {gauge }}^{\text {CPT-odd }}= & \left(k_{3}\right)_{\kappa} \epsilon^{\kappa \lambda \mu \nu} \operatorname{Tr}\left(G_{\lambda} G_{\mu \nu}+\frac{2}{3} i g_{3} G_{\lambda} G_{\mu} G_{\nu}\right) \\
& +\left(k_{2}\right)_{\kappa} \epsilon^{\kappa \lambda \mu \nu} \operatorname{Tr}\left(W_{\lambda} W_{\mu \nu}+\frac{2}{3} i g W_{\lambda} W_{\mu} W_{\nu}\right) \\
& +\left(k_{1}\right)_{\kappa} \epsilon^{\kappa \lambda \mu \nu} B_{\lambda} B_{\mu \nu}+\left(k_{0}\right)_{\kappa} B^{\kappa} .
\end{aligned}
$$

The coefficients $k_{1,2,3}$ are real and have dimensions of mass, while $k_{0}$ is also real and has dimensions of mass cubed. It turns out that, if any of these CPT-odd terms do indeed appear, they would generate instabilities in the minimal theory. They are all associated with negative contributions to the energy, and in addition the term with $k_{0}$ would directly generate a linear instability in the potential. It might therefore seem desirable that all the coefficients $k_{0,1,2,3}$ vanish. While this could be imposed at the classical level, radiative quantum corrections from, say, the fermion sector might $a$ priori be expected to generate nonzero values. Remarkably, the structure of the standard-model extension appears to be such that no corrections arise, at least to one loop. These issues are discussed further in what follows, in particular in Secs. IV A and V.

It is known that some apparently $C P T$ - and Lorentzviolating terms can be eliminated from the action via field 
redefinitions [2]. Several types of redefinition can be considered. In the context of the present standard-model extension, we have investigated a variety of possibilities for each field. As a general rule, the more complex the theoretical structure becomes, the less likely it is that a useful field redefinition exists. For instance, the presence of Lorentz-violating $C P T$-even derivative couplings in the standard-model extension complicates the analysis for $C P T$-odd terms provided in Ref. [2], although it turns out that the conclusions still hold. Here, we summarize a few methodological results and describe some special cases of particular interest.

To eliminate a Lorentz-breaking term, a field redefinition must involve the associated coupling coefficient. When derivative couplings play a role, the field redefinition may also involve spacetime-position variables. The assumption that the coupling coefficients are small can be helpful, in some cases directly assisting in derivations and in others leading to a set of approximate field redefinitions. Under the latter, a theory with first-order Lorentz-breaking effects may be redefined into one with effects appearing only at second or higher orders. Alternatively, some first-order Lorentz-breaking terms may be absorbed into others. A partial constraint on allowable redefinitions is provided by the transformation properties of the various Lorentz-violating terms under the discrete symmetries $C, P, T$. Only terms with identical discrete-symmetry properties can be absorbed into one another by first-order redefinitions.

Two types of redefinition that we have found of particular value are linear phase redefinitions and linear normalization redefinitions. For example, some terms involving the coefficients $a_{L, R, Q, U, D}$ can be eliminated by position-dependent field-phase redefinitions, as described in Ref. [2]. Another example is provided by terms involving the coefficients $H_{L, U, D}$, some of which can absorb through fieldnormalization redefinitions certain other terms involving the coefficients $c_{L, R, Q, U, D}$. These examples have specific interesting implications for the quantum-electrodynamics limit of the standard-model extension, and their explicit forms for that case are given in Sec. III below. Useful nonlinear field redefinitions might also exist in principle, but these are typically more difficult to implement meaningfully because they may represent (noncanonical) transformations between different physical systems rather than reinterpretations of the same physics.

We next consider the issue of electroweak $\mathrm{SU}(2) \times \mathrm{U}(1)$ symmetry breaking. The static potential for the gauge and Higgs fields can be extracted from the Lagrangian terms given above for the standard-model extension. It is possible to work in the unitary gauge as usual, since the Lorentzbreaking terms do not affect the gauge structure of the theory. The analysis is somewhat more complicated than the conventional case, as it involves additional terms depending on the coupling coefficients $k_{\phi \phi}, k_{\phi W}, k_{\phi}, k_{W}, k_{2}$, and $k_{0}$. In principle, there are also contributions from the SU(3) sector, but these decouple from the Higgs field and so the gluon expectation values can be taken to be zero as usual. As mentioned above, the terms $k_{2}$ and $k_{0}$ are expected to vanish for consistency of the minimal theory, and so we assume this in what follows. In fact, a nonzero $k_{2}$ would have no effect on the vacuum values of the fields, but the linear instability that would be introduced by a nonzero $k_{0}$ would exclude a stable vacuum in the absence of other (nonlinear) effects.

Extremizing the static potential produces five simultaneous equations. Three of these are satisfied if the expectation values of $W_{\mu}^{ \pm}$and the photon $A_{\mu}$ vanish. The other two equations can be solved algebraically for the expectation values of the Higgs and $Z_{\mu}^{0}$ fields. In the general case, both of these are nonzero and are given by

$$
\begin{gathered}
\left\langle Z_{\mu}^{0}\right\rangle=\frac{1}{q} \sin 2 \theta_{W}\left(\operatorname{Re} \hat{k}_{\phi \phi}\right)_{\mu \nu}^{-1} k_{\phi}^{\nu}, \\
\left\langle r_{\phi}\right\rangle=a\left(1-\frac{1}{\mu^{2}}\left(\operatorname{Re} \hat{k}_{\phi \phi}\right)_{\mu \nu}^{-1} k_{\phi}^{\mu} k_{\phi}^{\nu}\right)^{1 / 2},
\end{gathered}
$$

where $\hat{k}_{\phi \phi}^{\mu \nu} \equiv \eta^{\mu \nu}+k_{\phi \phi}^{\mu \nu}$ and $a \equiv \sqrt{6 \mu^{2} / \lambda}$. Note that the quantity $\left(\operatorname{Re} \hat{k}_{\phi \phi}\right)_{\mu \nu}^{-1}$ always exists when the Lorentz violation is small, $\left|\left(k_{\phi \phi}\right)^{\mu \nu}\right| \ll 1$. Note also that $\left\langle r_{\phi}\right\rangle$ is a scalar under both particle and observer Lorentz transformations, so quantities such as the fermion mass parameters remain scalars despite the presence of Lorentz breaking.

As might be anticipated, the above pattern of expectation values leaves unbroken the electromagnetic U(1) symmetry, and it can be shown that fluctuations about the extremum are stable. When substituted into the Lagrangian for the standard-model extension, the unconventional nonzero expectation value for the field $Z_{\mu}^{0}$ generates some additional $C P T$ - and Lorentz-violating contributions. However, these are all of the same form as other $C P T$ - and Lorentz-violating terms already present in the theory, so they can be absorbed into existing coupling coefficients.

Some analyses of experimental tests of the standardmodel extension involving flavor-changing oscillations in neutral mesons have been performed in Refs. [3,5,6]. Tests at the level of quantum electrodynamics are mentioned below. Note that some bounds on both the fermion and the gauge sectors might be obtained from available experimental information about the $Z_{\mu}^{0}$ and perhaps the $W_{\mu}^{ \pm}$. Such limits would be of interest in their own right, although it seems likely that they would be much weaker than required to detect suppressed Lorentz violation at the levels estimated in this work.

\section{EXTENDED QUANTUM ELECTRODYNAMICS}

In much the same way that conventional quantum electrodynamics (QED) can be obtained from the usual standard model, a generalized quantum electrodynamics incorporating Lorentz-breaking terms can be extracted from the standardmodel extension given in Sec. II. This is of particular interest because QED has been tested to high precision in a variety of experiments, some of which may tightly constrain the coupling coefficients of the possible Lorentz-violating terms.

A straightforward way to obtain the extended QED is as follows. After the $\mathrm{SU}(2) \times \mathrm{U}(1)$ symmetry breaking, set to zero the fields $G_{\mu}$ for the gluons, $W_{\mu}^{ \pm}, Z_{\mu}^{0}$ for the weak bosons, and the physical Higgs field (but not the expectation value of the Higgs doublet, which generates fermion 
masses). The only remaining boson is the photon, mediating the electromagnetic interactions. The neutrinos are charge neutral, so they decouple and can be discarded. The resulting theory is an extended QED describing the electromagnetic interactions of quarks and (charged) leptons. It is expected to inherit from the standard-model extension various attractive features mentioned in the introduction, including $\mathrm{U}(1)$ gauge invariance, energy-momentum conservation, observer Lorentz invariance, hermiticity, microcausality, positivity of the energy, and power-counting renormalizability.

Denote the standard four-component lepton fields by $l_{A}$ and their masses by $m_{A}$, where $A=1,2,3$ corresponds to electron, muon, tau, respectively. Then, the Lagrangian for the conventional QED of leptons and photons is

$$
\mathcal{L}_{\text {lepton-photon }}^{\text {QED }}=\frac{1}{2} i \bar{l}_{A} \gamma^{\mu} \overleftrightarrow{D}_{\mu} l_{A}-m_{A} \bar{l}_{A} l_{A}-\frac{1}{4} F_{\mu \nu} F^{\mu \nu} .
$$

In this equation and throughout what follows, $D_{\mu} \equiv \partial_{\mu}$ $+i q A_{\mu}$ and the field strength $F_{\alpha \beta}$ is defined by

$$
F_{\alpha \beta} \equiv \partial_{\alpha} A_{\beta}-\partial_{\beta} A_{\alpha},
$$

as usual.

The standard-model extension generates additional terms that violate Lorentz symmetry. The $C P T$-even terms involving the lepton fields are

$$
\begin{aligned}
\mathcal{L}_{\text {lepton }}^{\text {CPT-even }}= & -\frac{1}{2}\left(H_{l}\right)_{\mu \nu A B} \bar{l}_{A} \sigma^{\mu \nu} l_{B}+\frac{1}{2} i\left(c_{l}\right)_{\mu \nu A B} \bar{l}_{A} \gamma^{\mu} \overleftrightarrow{D}^{\nu} l_{B} \\
& +\frac{1}{2} i\left(d_{l}\right)_{\mu \nu A B} \bar{l}_{A} \gamma_{5} \gamma^{\mu} \overleftrightarrow{D}^{\nu} l_{B} .
\end{aligned}
$$

In this equation, the coupling coefficients $\left(H_{l}\right)_{\mu \nu A B}$ are antisymmetric in spacetime indices and have dimensions of mass. They arise from the coefficients in Eq. (13) following gauge-symmetry breaking, and they are Hermitian in generation space. The Hermitian dimensionless couplings $\left(c_{l}\right)_{\mu \nu A B}$ and $\left(d_{l}\right)_{\mu \nu A B}$ could in principle have both symmetric and antisymmetric spacetime components but can be taken as traceless. They arise from the expressions (9).

The $C P T$-odd terms involving the lepton fields are

$$
\mathcal{L}_{\text {lepton }}^{\text {CPT-odd }}=-\left(a_{l}\right)_{\mu A B} \bar{l}_{A} \gamma^{\mu} l_{B}-\left(b_{l}\right)_{\mu A B} \bar{l}_{A} \gamma_{5} \gamma^{\mu} l_{B} .
$$

The couplings $\left(a_{l}\right)_{\mu A B}$ and $\left(b_{l}\right)_{\mu A B}$ are Hermitian and have dimensions of mass. They arise from Eq. (10). Note that imposing individual lepton-number conservation in both the above equations would make all the coupling coefficients diagonal in flavor space.

In the pure-photon sector, there is one CPT-even Lorentz-violating term:

$$
\mathcal{L}_{\text {photon }}^{\text {CPT-even }}=-\frac{1}{4}\left(k_{F}\right)_{\kappa \lambda \mu \nu} F^{\kappa \lambda} F^{\mu \nu} .
$$

The coupling $\left(k_{F}\right)_{\kappa \lambda \mu \nu}$ arises from Eq. (16) and is real and dimensionless. Without loss of generality it can be taken as double traceless, since any trace component would serve merely to redefine the kinetic term and hence is just a field renormalization. We disregard a conceivable $\theta$-type term proportional to $F_{\kappa \lambda} \epsilon^{\kappa \lambda \mu \nu} F_{\mu \nu}$, which might arise from a totally antisymmetric component of $k_{F}$, on the grounds that it is a total derivative. The coupling $k_{F}$ therefore can be taken to have the symmetries of the Riemann tensor.

There is also a $C P T$-odd pure-photon term:

$$
\mathcal{L}_{\text {photon }}^{\text {CPT-odd }}=+\frac{1}{2}\left(k_{A F}\right)^{\kappa} \epsilon_{\kappa \lambda \mu \nu} A^{\lambda} F^{\mu \nu},
$$

where the coupling coefficient $\left(k_{A F}\right)^{\kappa}$ is real and has dimensions of mass. This term arises from the $C P T$-odd gauge sector (17) of the standard-model extension. As mentioned in the previous section, it has some theoretical difficulties associated with negative contributions to the energy and it therefore seems likely to be absent in practice. It is included in what follows so that we can discuss explicitly its difficulties and some related issues involving radiative corrections. Note also that the excluded destabilizing linear term in $B_{\mu}$ in the standard-model extension would, if present, generate a corresponding linear term $-\left(k_{A}\right)_{\kappa} A^{\kappa}$ in Eq. (25), where $\left(k_{A}\right)_{\kappa}$ is a real coupling with dimensions of mass cubed. Certain issues involving this term are addressed in Secs. IV A and V.

The QED limit obtained from the standard-model extension also has a quark sector. This has the same general form as the lepton sector given by Eqs. (20), (22), and (23), except that six quark fields replace the three leptons and so twice as many Lorentz-violating couplings occur. Note that the lepton and quark sectors are coupled only through the photon: the gauge invariance of the standard-model extension excludes couplings mixing leptons and quarks.

The extended QED of leptons and photons given in Eqs. (20)-(25) should suffice for certain applications where the asymptotic states are leptons or photons and the strong and weak interactions play a negligible role, including a variety of existing or proposed high-precision experiments involving leptons. Interesting options for such experiments are to establish the possible signals of Lorentz violation suggested by the extended QED and to place bounds on the associated coupling coefficients. For example, promising possibilities involving the muon include accurate measurements of $g-2$ such as those underway at the Brookhaven muon ring [20] and sensitive tests for the decay $\mu \rightarrow e \gamma$. There are also a variety of other comparisons involving heavy leptons that are potentially of interest [21]. These issues lie beyond the scope of the present work and will be addressed elsewhere.

For certain experiments, it suffices to consider another limiting case of the theory: the extended QED including only electrons, positrons and photons. This limit can be extracted from the Lagrangian terms for the extended QED of leptons and photons by setting to zero the muon and the $\tau$ fields. Denoting the four-component electron field by $\psi$ and the electron mass by $m_{e}$, the usual QED Lagrangian for electrons and photons is

$$
\mathcal{L}_{\text {electron }}^{\mathrm{QED}}=\frac{1}{2} i \bar{\psi} \gamma^{\mu} \stackrel{\leftrightarrow}{D}_{\mu} \psi-m_{e} \bar{\psi} \psi-\frac{1}{4} F_{\mu \nu} F^{\mu \nu}
$$

In the Lorentz-violating sector, the pure-photon terms are still given by Eqs. (24) and (25). However, the CPT-even terms in the fermion sector become 


$$
\begin{aligned}
\mathcal{L}_{\text {electron }}^{\text {CPT-even }}= & -\frac{1}{2} H_{\mu \nu} \bar{\psi} \sigma^{\mu \nu} \psi+\frac{1}{2} i c_{\mu \nu} \bar{\psi} \gamma^{\mu} \overleftrightarrow{D}^{\nu} \psi \\
& +\frac{1}{2} i d_{\mu \nu} \bar{\psi} \gamma_{5} \gamma^{\mu} \overleftrightarrow{D}^{\nu} \psi,
\end{aligned}
$$

while the $C P T$-odd ones become

$$
\mathcal{L}_{\text {electron }}^{\text {CPT-odd }}=-a_{\mu} \bar{\psi} \gamma^{\mu} \psi-b_{\mu} \bar{\psi} \gamma_{5} \gamma^{\mu} \psi
$$

The real coupling coefficients $a, b, c, d$, and $H$ are the $(1,1)$-flavor components of the corresponding coefficients in the extended QED of leptons and photons and inherit the corresponding dimensions and Lorentz-transformation properties.

In addition to the expressions given in Eqs. (24)-(28) for the extended QED of electrons, positrons, and photons, other Lorentz-violating terms can be envisaged that are compatible with $U(1)$ charge symmetry, renormalizability, and an origin in spontaneous Lorentz breaking but that cannot be obtained as a reduction from the standard-model extension. All such terms would be $C P T$ odd. They would have the form

$$
\mathcal{L}_{\text {electron }}^{\text {extra }}=\frac{1}{2} i e_{\nu} \bar{\psi} \overleftrightarrow{D}^{\nu} \psi-\frac{1}{2} f_{\nu} \bar{\psi} \gamma_{5} \overleftrightarrow{D}^{\nu} \psi+\frac{1}{4} i g_{\lambda \mu \nu} \bar{\psi} \sigma^{\lambda \mu} \overleftrightarrow{D}^{\nu} \psi,
$$

where the couplings $e_{\mu}, f_{\mu}$, and $g_{\lambda \mu \nu}$ are real and dimensionless. The reason such terms are absent from the expressions obtained above is that all putative renormalizable terms in the standard-model extension that could generate Eq. (29) are directly incompatible with the electroweak structure. However, it is possible that nonrenormalizable higherdimensional operators in the effective Lagrangian obeying $\mathrm{SU}(2) \times \mathrm{U}(1)$ symmetry and involving the Higgs field might generate the expressions (29) when the Higgs field acquires its vacuum expectation value. According to standard lore and the discussion in Sec. II, such operators would be expected to be highly suppressed relative to those we have listed for the standard-model extension. This suppression should remain in force at the level of the extended QED, which means any terms of the form (29) would be expected to have coupling coefficients much smaller than the other terms we consider. Similar considerations apply to possible extra terms that might appear in the heavy-lepton and quark sectors of the extended QED.

Next, we address the issue of field redefinitions within the context of the extended QED of electrons, positrons, and photons. We have found several cases to be especially useful. One is a linear phase redefinition of the form $\psi$ $=\exp (-i a \cdot x) \chi$, which eliminates the term $-a_{\mu} \bar{\psi} \gamma^{\mu} \psi$ from Eq. (28). This is equivalent to shifting the zeros of energy and momentum for electrons and positrons [2]. We therefore expect no observable effects from a nonzero $a_{\mu}$ in any QED experiment.

Another useful class of redefinitions involves field renormalizations depending on coupling coefficients. For a fermion field $\psi$, consider the redefinition

$$
\psi=(1+v \cdot \Gamma) \chi,
$$

where $\Gamma$ is one of $\gamma^{\mu}, \gamma_{5} \gamma^{\mu}, \sigma^{\mu \nu}$ and $v$ is a combination of coupling coefficients with appropriate spacetime indices.
This set of redefinitions can be used to obtain several useful approximate results, valid to first order in the (small) coupling coefficients. One is that the combination $\epsilon^{\mu \nu \alpha \beta} H_{\alpha \beta}$ $+m\left(d^{\mu \nu}-d^{\nu \mu}\right)$ can be eliminated and hence is unobservable at leading order in any QED experiment. Only the orthogonal linear combination is physical at this level. Another is that the antisymmetric component of $c_{\mu \nu}$ can be eliminated to first order. Similarly, even if the extra terms with coefficients $e_{\mu}$ and $f_{\mu}$ in Eq. (29) should appear, they could be eliminated to first order by a combination of field redefinitions. The same is true of the trace components of the extra term with coefficient $g_{\lambda \mu \nu}$, while the totally antisymmetric component of this term can be absorbed into $b_{\mu}$ to first order. Combining all these results, it follows that at leading order in the extended QED of electrons, positrons, and photons the only observable coupling coefficients can be taken as $b_{\mu}, H_{\mu \nu}$, the symmetric components of $c_{\mu \nu}$ and $d_{\mu \nu}$, and possibly the traceless mixed-symmetry components of the extra coefficient $g_{\lambda \mu \nu}$.

So far in this section we have considered various forms of extended QED that emerge as limits of the standard-model extension. For some purposes, it can be useful to work within an effective extended QED valid for a free fermion that is a composite of leptons and quarks, such as a nucleon, atom, or ion. For a single fermion field $\psi$ of this type, the effective Lagrangian would then have the same form as that of the extended QED for electrons, positrons, and photons. A description of this type is useful for investigations of the implications of high-precision experiments on composite fermions, such as comparative tests of proton properties or searches for a neutron electric-dipole moment. In principle, extra terms of the form (29) could appear as a result of the interactions among the fermion constituents, but in the effective theory the coupling coefficients of such terms would involve combinations of the constituent coupling coefficients with the interaction coupling constants and might therefore be expected to be absent at leading order in many cases.

Some possible experimental signals from extended QED are investigated in Ref. [8]. Certain high-precision tests that could be performed with present technology are considered, and the attainable bounds on Lorentz-breaking coupling coefficients are estimated. The tests involve comparative measurements of anomalous magnetic moments and charge-tomass ratios for particles and antiparticles confined in a Penning trap [7]. They typically have the potential to bound the coupling coefficients of Lorentz- and $C P T$-violating terms at a level close to that expected from Planck-scale suppression. For example, the spacelike components of the coefficient $b_{\mu}$ control the appropriate figure of merit for experiments comparing the anomalous magnetic moments of the electron and positron. This figure of merit can be bounded to about one part in $10^{20}$, which is comparable to the ratio of $m_{e} / M$ of the electron mass to the Planck scale.

\section{THE PURE-PHOTON SECTOR}

In this section, we focus on the pure-photon sector of the extended QED. We examine some theoretical implications of 
the existence of Lorentz- and $C P T$-violating terms and address some experimental issues.

\section{A. Lagrangian and energy-momentum tensor}

The Lagrangian of interest, which is $\mathrm{U}(1)$ gauge invariant by construction, is a combination of the photon term in Eq. (26) with the expressions (24) and (25). It is

$$
\begin{aligned}
\mathcal{L}_{\text {photon }}^{\text {total }}= & -\frac{1}{4} F_{\mu \nu} F^{\mu \nu}-\frac{1}{4}\left(k_{F}\right)_{\kappa \lambda \mu \nu} F^{\kappa \lambda} F^{\mu \nu} \\
& +\frac{1}{2}\left(k_{A F}\right)^{\kappa} \epsilon_{\kappa \lambda \mu \nu} A^{\lambda} F^{\mu \nu}
\end{aligned}
$$

Some properties of the coupling coefficients $k_{F}$ and $k_{A F}$ are described following Eqs. (24) and (25). For certain calculations, it is useful to decompose the coefficient $k_{F}$ into its two Lorentz-irreducible pieces, one with 10 independent components analogous to the Weyl tensor in general relativity and one with nine components analogous to the trace-free Ricci tensor. Only one of the 19 total independent components of $k_{F}$ (the 00 component of the trace-free Ricci-tensor analog) and one of the four independent components of the coefficient $k_{\mathrm{AF}}$ (the timelike component $k_{A F}^{0}$ ) are associated with terms invariant under (particle) rotations.

Some insight into the structure of the Lagrangian can be obtained by expressing it in terms of the potentials $\phi, \vec{A}$ and the fields $\vec{E}, \vec{B}$. We find

$$
\begin{aligned}
\mathcal{L}_{\text {photon }}^{\text {total }=} & \frac{1}{2}\left(\vec{E}^{2}-\vec{B}^{2}\right)+\frac{1}{2} \alpha\left(\vec{E}^{2}+\vec{B}^{2}\right)+\frac{1}{2} \beta_{E}^{j k} E^{j} E^{k} \\
& +\frac{1}{2} \beta_{B}^{j k} B^{j} B^{k}+\frac{1}{2} \beta_{E B}^{j k} E^{j} B^{k}+k_{A F}^{0} \vec{A} \cdot \vec{B} \\
& -\phi \vec{k}_{A F} \cdot \vec{B}+\vec{k}_{A F} \cdot(\vec{A} \times \vec{E}) .
\end{aligned}
$$

Here and throughout this work, $j, k, \ldots=1,2,3$ are spatial indices. The real coefficients $\alpha$ and $\beta_{E}^{j k}, \beta_{B}^{j k}, \beta_{E B}^{j k}$ are various combinations of the couplings $\left(k_{F}\right)_{\kappa \lambda \mu \nu}$ appearing in Eq. (31). Disregarding as before any total-derivative effects, the $\beta_{E}^{j k}, \beta_{B}^{j k}, \beta_{E B}^{j k}$ are traceless. Note that all possible quadratic combinations of the electric and magnetic fields appear. Only two terms, involving $\alpha$ and $k_{A F}^{0}$, preserve (particle) rotational invariance. Note also that a rescaling without physical consequences can be performed to obtain a standard normalization of the electric field $\vec{E}$. This produces a Lagrangian of the same general form as Eq. (32) except that the Lorentzbreaking term proportional to $\left(\vec{E}^{2}+\vec{B}^{2}\right)$ is replaced with one proportional to $\vec{B}^{2}$ alone.

The canonical energy-momentum tensor can be constructed following the standard procedure. This tensor can be partially symmetrized, but complete symmetrization is impossible because there is an antisymmetric component that cannot be written as a total derivative. A relatively elegant expression can be obtained by adding judiciously chosen total-derivative terms, which leave unchanged the physics. Denoting the resulting energy-momentum tensor by $\Theta^{\mu \nu}$, we find

$$
\begin{aligned}
\Theta^{\mu \nu}= & -F^{\mu \gamma} F_{\gamma}^{\nu}+\frac{1}{4} \eta^{\mu \nu} F_{\alpha \beta} F^{\alpha \beta}-\left(k_{F}\right)^{\alpha \beta \mu \gamma} F^{\nu}{ }_{\gamma} F_{\alpha \beta} \\
& +\frac{1}{4} \eta^{\mu \nu}\left(k_{F}\right)_{\alpha \beta \gamma \delta} F^{\alpha \beta} F^{\gamma \delta}+\left(k_{A F}\right)^{\nu} A_{\alpha} \widetilde{F}^{\alpha \mu} .
\end{aligned}
$$

Here, we define

$$
\widetilde{F}^{\mu \nu}=\epsilon^{\kappa \lambda \mu \nu} F_{\kappa \lambda} / 2
$$

to be the dual field strength.

The energy-momentum tensor obeys the usual conservation relation,

$$
\partial_{\mu} \Theta^{\mu \nu}=0
$$

In addition to the gauge-invariant and symmetric contributions to $\Theta^{\mu \nu}$, which include the conventional pieces among others, there are additional terms involving the coefficient $k_{F}$ that are gauge invariant but asymmetric. The term with $k_{A F}$ is neither gauge invariant nor symmetric. Under a gauge transformation, an additional total-derivative term appears. The presence of an antisymmetric component in $\Theta^{\mu \nu}$ implies that care is required in physical interpretations of the energymomentum behavior. Although $\Theta^{j 0}$ can be regarded as the components of a generalized Poynting vector, its volume integral is no longer conserved and cannot be identified with the conserved volume integral of the components $\Theta^{0 j}$ of the momentum density. These features are a direct consequence of the presence of the background expectation values of tensor fields, represented in the low-energy theory by the coupling coefficients $k_{F}$ and $k_{A F}$.

The energy density is given by the component $\Theta^{00}$. Inspection shows it can be written in the form

$$
\begin{aligned}
\Theta^{00}= & \frac{1}{2}\left(\vec{E}^{2}+\vec{B}^{2}\right)-\left(k_{F}\right)^{0 j 0 k} E^{j} E^{k} \\
& +\frac{1}{4}\left(k_{F}\right)^{j k l m} \epsilon^{j k p} \epsilon^{l m q} B^{p} B^{q}-\left(k_{A F}\right)^{0} \vec{A} \cdot \vec{B} .
\end{aligned}
$$

If $k_{A F}$ vanishes and $k_{F}$ is small, $\Theta^{00}$ is nonnegative. This can be seen as follows. The combination of the usual energy density with the terms proportional to $k_{F}$ can be viewed as a bilinear form $x^{T} M x$ generated from a matrix $M$ in the sixdimensional space $x^{T} \equiv(\vec{E}, \vec{B})$. The matrix $M$ is symmetric and $3 \times 3$-block diagonal, since no cross terms in $\vec{E}$ and $\vec{B}$ appear in $\Theta^{00}$. Observer rotation invariance can be used to diagonalize the upper $3 \times 3$ block associated with the electric field. Since $k_{F}$ is small, the three diagonal entries are of the form $\frac{1}{2}-O\left(k_{F}\right)>0$, so the contribution to $\Theta^{00}$ from the electric field is non-negative in any frame. A similar argument shows that the contribution from the lower $3 \times 3$ block, associated with the magnetic field, is also non-negative. The conserved energy $\mathcal{E}$ of a field configuration, obtained by integrating $\Theta^{00}$ over all space, is therefore also non-negative.

If instead $k_{F}$ vanishes and $k_{A F}$ is small, the contribution to $\mathcal{E}$ can be written in the form

$$
\begin{aligned}
\mathcal{E} & \equiv \int d^{3} x \Theta^{00} \\
& =\frac{1}{2} \int d^{3} x\left(\vec{E}^{2}+\left[\vec{B}-\left(k_{A F}\right)^{0} \vec{A}\right]^{2}-\left[\left(k_{A F}\right)^{0}\right]^{2} \vec{A}^{2}\right) .
\end{aligned}
$$


The last term is nonpositive and so can, in principle, introduce an instability in the theory [22]. Note that a similar situation would hold for the linear term $-\left(k_{A}\right)_{\kappa} A^{\kappa}$ in the Lagrangian that was discarded in Sec. III, for which the energy density $\left(k_{A}\right)_{0} \phi-\vec{k}_{A} \cdot \vec{A}$ could also be negative. The appearance of negative contributions to the energy is unsatisfactory from a theoretical viewpoint.

It might seem tempting to resolve this issue by requiring that only the spacelike components of $k_{A F}$ are nonzero, so that the terms involving $\left(k_{A F}\right)^{0}$ are absent from Eqs. (36) and (37). However, this condition depends on the observer frame, so even an infinitesimal boost to another observer frame would reintroduce the instability. A somewhat more interesting option might be to combine the vanishing of $\left(k_{A F}\right)^{0}$ with the introduction of a (small) photon mass, perhaps arising from a hitherto unobserved spontaneous breaking of the electromagnetic U(1) gauge symmetry. This would eliminate the linear instability and in principle might also produce a contribution canceling the negative term appearing in Eq. (37), although perhaps only for a physically reasonable range of observer boosts determined by the size of the photon mass and the magnitude of the components of $\vec{k}_{A F}$. Although some form of this idea might be made physically acceptable, we are restricting ourselves here to minimal modifications of the usual standard model and so we disregard this possibility in the present work.

In the absence of a complete demonstration of a consistent alternative interpretation, one option might be to discard the term (25) depending on $k_{A F}$. This is possible at the classical level, but at the quantum level one might expect radiative corrections to induce it. We return to this question in Sec. V, meanwhile keeping the term (25) in the analysis for completeness.

\section{B. Solution of equations of motion}

The equations of motion arising from the Lagrangian (31) are

$$
\partial_{\alpha} F_{\mu}{ }^{\alpha}+\left(k_{F}\right)_{\mu \alpha \beta \gamma} \partial^{\alpha} F^{\beta \gamma}+\left(k_{A F}\right)^{\alpha} \epsilon_{\mu \alpha \beta \gamma} F^{\beta \gamma}=0 .
$$

These equations are the Lorentz-breaking extensions of the usual inhomogeneous Maxwell equations in the absence of sources, $\partial_{\mu} F^{\mu \nu}=0$. By virtue of its conventional definition in Eq. (21), the field strength $F^{\mu \nu}$ satisfies the usual homogeneous Maxwell equations

$$
\partial_{\mu} \widetilde{F}^{\mu \nu}=0,
$$

where $\widetilde{F}^{\mu \nu}$ is given in Eq. (34).

An important feature of Eqs. (38) and (39) is their linearity in $F_{\mu \nu}$ and hence in $A_{\mu}$. The Lorentz-violating terms thereby avoid the complications of nonlinear modifications to the Maxwell equations, which are known to occur in some physical situations such as nonlinear optics or when vacuumpolarization effects are included. Another feature is that the extra Lorentz-violating terms involve both the electric and the magnetic fields, as well as their derivatives. As a result, Eqs. (38) bear some resemblance to the usual Maxwell equa- tions in moving media, for which the boost causes the electric and magnetic fields to mix. Note that the coefficients determining this mixing are directly dependent on the velocity of the medium and so change with the inertial frame. Similarly, for the Lorentz-violating case of interest here, a change of observer frame changes the coupling coefficients. Some other useful analogies between Eqs. (38) and those of conventional electrodynamics in macroscopic media are described in Sec. IV C.

The equations of motion (38) and (39) depend only on $F_{\mu \nu}$ and so, as expected, they are gauge invariant under the standard $\mathrm{U}(1)$ gauge transformations

$$
A_{\mu} \rightarrow A_{\mu}-\frac{1}{q} \partial_{\mu} \Lambda
$$

As in conventional electrodynamics, the presence of gauge symmetry affects the interpretation and solution of the equations of motion. We first consider a treatment in terms of the potentials $A_{\mu}$ and then one for the field strengths $\vec{E}, \vec{B}$.

Taking the potentials $A_{\mu}$ as basic, the four equations (39) are directly satisfied. This appears to leave four equations (38) for four unknowns $A_{\mu}$. However, just as in conventional Maxwell electrodynamics, the conjugate momentum to $A_{0}$ vanishes because $\partial_{0} A^{0}$ is missing from the Lagrangian (31), so the theory has a Dirac primary constraint. In the conventional case it then follows from the identity $\partial_{\nu} \partial_{\mu} F^{\mu \nu} \equiv 0$, which is associated with current conservation when sources are present, that the equation of motion associated with $A_{0}$ plays the role of an initial condition. The same conclusion holds here because when acted on by $\partial_{\mu}$ the left-hand side of Eq. (38) also vanishes identically. This leaves three equations of motion and a constraint for four variables. One combination of variables can be fixed by a gauge choice. The constraint then leaves two independent degrees of freedom.

Despite the parallels with conventional electrodynamics, the gauge-fixing process involves some interesting differences. For example, there is normally an equivalence between the Coulomb gauge $\vec{\nabla} \cdot \vec{A}=0$, the temporal gauge $A^{0}$ $=0$, and one of the members of the family of Lorentz gauges $\partial_{\mu} A^{\mu}=0$. When Lorentz-violating effects are included, these three gauge choices become inequivalent. For example, $A^{0}$ typically is nonzero if the Coulomb gauge $\vec{\nabla} \cdot \vec{A}=0$ is imposed.

More insight about the wave motion implied by Eq. (38) can be gained with the ansatz

$$
A_{\mu}(x) \equiv \epsilon_{\mu}(p) \exp \left(-i p_{\alpha} x^{\alpha}\right),
$$

where $p^{\mu} \equiv\left(p^{0}, \vec{p}\right)$ can be regarded as the frequency and wave vector of the mode or as the associated energy and momentum (which can be distinct from the conserved energy and momentum obtained from the energy-momentum tensor). Note that taking the real part is understood, as usual. The equations of motion (38) generate the momentum-space equation

$$
M^{\alpha \delta}(p) A_{\delta}=0
$$


where the matrix $M^{\alpha \delta}(p)$ is

$$
\begin{aligned}
M^{\alpha \delta}(p) \equiv & \eta^{\alpha \delta} p^{2}-p^{\alpha} p^{\delta}-2\left(k_{F}\right)^{\alpha \beta \gamma \delta} p_{\beta} p_{\gamma} \\
& -2 i\left(k_{A F}\right)_{\beta} \epsilon^{\alpha \beta \gamma \delta} p_{\gamma} .
\end{aligned}
$$

This $4 \times 4$ complex-valued matrix is Hermitian because the first three terms are real and symmetric while the last is imaginary and antisymmetric. Its determinant can be shown to vanish identically for all $p^{\mu}$, a feature related to the gauge freedom. The conventional result is recovered when the coefficients $k_{F}$ and $k_{A F}$ vanish.

Once a gauge choice is imposed, relation (42) provides a set of complex-valued equations for $A_{\delta}$. The differences between various gauge choices that normally are equivalent can be seen explicitly at this stage. For example, $M^{\alpha \delta}(p) A_{\delta}$ is not proportional to $A^{\alpha}$ in the Lorentz gauges, the Coulomb gauge leaves a nontrivial equation for $A^{0}$, and the temporal gauge generates an involved constraint on $\vec{\nabla} \cdot \vec{A}$. In practice, Eq. (42) then reduces to a (sub)set of equations involving an effective matrix $M_{\text {eff }}(p)$ with the explicit form dependent on the gauge choice. The requirement for the existence of nonzero solutions can be obtained from a condition of the type $\operatorname{det} M_{\text {eff }}(p)=0$. With fixed coefficients $k_{F}$ and $k_{A F}$, this condition then determines $p^{0}$ as a function of $\vec{p}$. Since $M^{\mu \alpha}(p)$ is a $4 \times 4$ matrix with entries quadratic in $p^{\mu}$, a determinant of this type can produce an eighth-order polynomial in $p^{0}$.

In the conventional case in the Lorentz gauge, the polynomial reduces to one with two quadruply degenerate roots, $p^{0}= \pm|\vec{p}|$. The apparent doubling of the roots relative to the number of variables can be understood by the observation that in this case $M^{\mu \alpha}(p)$ is symmetric under $p^{\mu} \rightarrow-p^{\mu}$, so for each solution $p^{0}(\vec{p})$ there is another solution $-p^{0}(-\vec{p})$. These two solutions can be shown to be physically equivalent by examining the real part of $A_{\mu}$ in Eq. (41).

In contrast, in the general extended electrodynamics the polynomial determining $p^{0}$ may have eight distinct roots. Each of these could in principle produce a nontrivial solution for $A_{\delta}$, double the expected number. In this case, $M^{\mu \alpha}(p)$ is symmetric under the simultaneous operations $p^{\mu} \rightarrow-p^{\mu}$ and $k_{A F} \longrightarrow-k_{A F}$ (leaving $k_{F}$ unchanged). Thus, for each solution $p^{0}\left(\vec{p}, k_{F}, k_{A F}\right)$ there is another solution $-p^{0}\left(-\vec{p}, k_{F}\right.$, $\left.-k_{A F}\right)$. The sign change for the coefficient $k_{A F}$ might appear to preclude the demonstration of the physical equivalence of these solutions. However, hermiticity of $M^{\mu \alpha}(p)$ implies its determinant is equivalent to the determinant of its complex conjugate. Since $k_{A F}$ appears only in the imaginary part of $M^{\mu \alpha}(p)$, it follows that for each solution $-p^{0}\left(-\vec{p}, k_{F}\right.$, $\left.-k_{A F}\right)$ there is also a solution $-p^{0}\left(-\vec{p}, k_{F},+k_{A F}\right)$. This is physically equivalent to the solution $p^{0}\left(\vec{p}, k_{F}, k_{A F}\right)$, as before. Thus, the number of independent roots is the same as the number of variables as expected, despite the apparent complexity of the polynomial.

In the general extended electrodynamics, neither the Coulomb gauge nor the Lorentz gauges significantly simplify the primary constraint. In contrast, the temporal gauge $A^{0}=0$ immediately removes one degree of freedom from $A_{\mu}$. This gauge can be imposed by choosing the function $\Lambda$ in Eq. (40) as $q \Lambda(t, \vec{x})=\int{ }^{t} A^{0}\left(t^{\prime}, \vec{x}\right) d t^{\prime}$. Note that this choice breaks ob- server boost invariance but leaves unaffected the observer rotation invariance. It reduces the primary constraint to the form

$$
M^{0 j} A^{j}=0
$$

where $M^{0 j}$ are components in the temporal gauge of the matrix $M^{\alpha \delta}$ given in Eq. (43).

At this stage an explicit solution could be found. For example, one could use two of the degrees of freedom of observer rotation invariance to select a convenient coordinate system, such as one in which $p^{j} \equiv(0,0, p)$. Solving for $A^{3}$ from the primary constraint (44) and substituting into the remaining three equations of motion in Eq. (42) would then produce an identity and two simultaneous linear equations for $A^{1}$ and $A^{2}$. A nontrivial solution of this pair of equations could be found by requiring the determinant of the system to vanish, which in turn would generate a relation between $p^{0}$ and $p$. Solving this relation must give two independent dispersion relations, one for each of the two physical degrees of freedom. The full dispersion relations in an arbitrary coordinate system could then in principle be recovered by using arguments based on rotational covariance.

Rather than pursuing this approach, we return to the eight equations of motion (38) and (39) and reconsider their treatment taking as independent variables the six electric and magnetic fields. Here, we are interested in the properties of electromagnetic radiation, so we work with the standard ansatz

$$
F_{\mu \nu}(x) \equiv F_{\mu \nu}(p) \exp \left(-i p_{\alpha} x^{\alpha}\right),
$$

where $p^{\mu} \equiv\left(p^{0}, \vec{p}\right)$.

Equations (39), which include the usual Faraday law and the condition ensuring the absence of magnetic monopoles, are unaffected by the Lorentz breaking and for radiation reduce as usual to

$$
p^{0} \vec{B}=\vec{p} \times \vec{E}, \quad \vec{p} \cdot \vec{B}=0 .
$$

The first of these can be regarded as defining the magnetic field once the electric field is known. The second of these equations follows from the first, and shows that the magnetic field remains transverse to $\vec{p}$ despite the Lorentz violation.

Equations (38) generate modified Coulomb and Ampère laws that are to be solved for $\vec{E}$. A relatively straightforward procedure is to substitute for $\vec{B}$ from Eq. (46). Using the Ampère law, we thereby obtain the vector equation

$$
M^{j k} E^{k}=0
$$

where the $3 \times 3$ matrix $M^{j k}$ is identical to the ( $j k)$-component submatrix of the matrix $M^{\alpha \delta}$ in Eq. (43). The modified Coulomb law can be obtained from the modified Ampère law by taking the scalar product with $\vec{p}$ : $p^{j} M^{j k} E^{k}=0$. Note that this derivation provides some insight about the temporal gauge $A^{0}=0$ in a treatment using $A_{\mu}$. Thus, there is a close parallel between the two because $\vec{E}$ $=i p^{0} \vec{A}$ in this gauge. 
To obtain an explicit solution, it is helpful to take advantage of the observer rotation invariance to select a coordinate system in which key expressions are simplified. For example, a useful frame is the one with $\vec{p}=(0,0, p)$. In it, the modified Coulomb equation can be solved for $E^{3}$ in terms of $E^{1}$ and $E^{2}$. Substitution of this solution into the three component equations (47) produces one identity and two simultaneous linear equations for $E^{1}$ and $E^{2}$. The matrix of this system of equations is Hermitian, and the condition for a nontrivial solution is that its determinant vanishes. The determinant turns out to be a fourth-order polynomial for $p^{0}$ as a function of $p$. For reasons similar to those given for the $A_{\mu}$ case, there are only two physically distinct solutions of this polynomial, one for each of the two independent degrees of freedom.

An explicit solution of the determinant condition in the general case is involved because the fourth-order polynomial is homogeneous in the small coupling coefficients. Since $k_{F}$ is dimensionless while $k_{A F}$ has dimensions of mass, to first order in the coupling coefficients and for $p^{0} \approx p \gg\left|k_{A F}\right|$ and $1 \gg\left|k_{F}\right|$ the solution for $p^{0}$ as a function of $p$ must take the form of the sum of $p$ with a function of the quantities $k_{F} p$ and $k_{A F}$. Indeed, we find

$$
p^{0}=(1+\rho) p \pm \sqrt{\left(\sigma^{2} p^{2}+\tau^{2}\right)},
$$

where $\rho$ and $\sigma^{2}$ are functions of the components of $k_{F}$ and $\tau^{2}$ is a function of the components of $k_{A F}$, given by

$$
\begin{aligned}
\rho= & \frac{1}{2}\left[\left(k_{F}\right)^{0101}+\left(k_{F}\right)^{0202}+\left(k_{F}\right)^{1313}\right. \\
& \left.+\left(k_{F}\right)^{2323}+2\left(k_{F}\right)^{0113}+2\left(k_{F}\right)^{0223}\right], \\
\sigma^{2}= & \frac{1}{4}\left[\left(k_{F}\right)^{0101}-\left(k_{F}\right)^{0202}+\left(k_{F}\right)^{1313}\right. \\
& \left.-\left(k_{F}\right)^{2323}+2\left(k_{F}\right)^{0113}-2\left(k_{F}\right)^{0223}\right]^{2} \\
& +\left[\left(k_{F}\right)^{0102}-\left(k_{F}\right)^{0123}+\left(k_{F}\right)^{0213}+\left(k_{F}\right)^{1323}\right]^{2} \\
\tau^{2}= & {\left[\left(k_{A F}\right)^{3}-\left(k_{A F}\right)^{0}\right]^{2} . }
\end{aligned}
$$

The solution (48) entangles the components of $k_{F}$ and $k_{A F}$ in a way that cannot be separated without additional information about their relative sizes. Note that it reduces correctly to the result of Ref. [22] in the case $k_{F}=0$.

The corresponding general solutions for the vectors of the electric and magnetic fields are involved and provide little insight for present purposes, so we omit them here. They exhibit two physical linear polarization vectors for $\vec{E}$, each obeying a different dispersion relation. This produces birefringence, among other effects. Note in particular that, contrary to widespread assumption in the literature, no circularly polarized solution to the equations of motion typically exists. An electromagnetic wave prepared in a state of circular polarization would propagate as two linearly polarized components with distinct dispersion relations, so an initial circularly polarized configuration would gradually become elliptical. These and some other interesting results about the wave propagation are discussed further in Secs. IV C and IV D below.
In the remainder of this subsection, we present a sample analytical solution to the equations of motion for a special case that provides further insight. We consider the Lagrangian (31) with $\left(k_{A F}\right)^{\mu}=0$ and with the only nonzero components of $k_{F}$ chosen to be $\left(k_{F}\right)_{0 j 0 k}=-\frac{1}{2} \beta_{j} \beta_{k}$, where the $\beta^{j}$ are three (small) real dimensionless quantities, and components related to these by the symmetries of $k_{F}$. In terms of the Lagrangian (32) only the term involving $\beta_{E}^{j k}$ is nonzero, and it has a direct-product structure: $\beta_{E}^{j k} \equiv+\beta^{j} \beta^{k}$. The Lagrangian (32) therefore becomes

$$
\mathcal{L}_{\text {photon }}^{\text {special }}=\frac{1}{2}\left(\vec{E}^{2}-\vec{B}^{2}\right)+\frac{1}{2}(\vec{\beta} \cdot \vec{E})^{2} .
$$

This example involves only $C P T$-even Lorentz violation.

The Lagrangian (50) generates modified inhomogeneous Maxwell equations in the absence of sources:

$$
\begin{aligned}
\vec{\nabla} \cdot \vec{E} & =-\vec{\beta} \cdot \vec{\nabla}(\vec{\beta} \cdot \vec{E}), \\
\vec{\nabla} \times \vec{B}-\partial_{0} \vec{E} & =\vec{\beta} \partial_{0}(\vec{\beta} \cdot \vec{E}) .
\end{aligned}
$$

In terms of the potentials $A_{\mu}$ of Eq. (41), appropriate for describing radiation in momentum space, these are equivalent to the vector equation

$$
\begin{gathered}
p^{0}[\vec{p}+(\vec{p} \cdot \vec{\beta}) \vec{\beta}] A^{0}-\left(p^{0}\right)^{2}[\vec{A}+(\vec{A} \cdot \vec{\beta}) \vec{\beta}] \\
+\left[\vec{p}^{2} \vec{A}-(\vec{p} \cdot \vec{A}) \vec{p}\right]=0
\end{gathered}
$$

and its scalar product with $\vec{p}$.

For definiteness we proceed in Lorentz gauge, where $A^{0}$ $=\vec{p} \cdot \vec{A} / p^{0}$. According to the discussions above, in the presence of Lorentz violation this gauge may require nonzero $A^{0}$ and $\vec{p} \cdot \vec{A}$. For a nontrivial solution to Eq. (52), we find two possible dispersion relations:

$$
\begin{aligned}
& \left(p_{o}\right)^{2}=0, \\
& \left(p_{e}\right)^{2}=-\frac{\left(\vec{\beta} \times \vec{p}_{e}\right)^{2}}{1+\vec{\beta}^{2}} .
\end{aligned}
$$

The first corresponds to an "ordinary" mode with fourmomentum $p_{o}$ obeying the conventional dispersion relation, while the second is an "extraordinary" mode with fourmomentum $p_{e}$ and a modified dispersion relation.

For a wave vector aligned along $\vec{\beta}$, both modes reduce to the conventional case and exhibit normal behavior. However, for other alignments the properties of the two modes differ. For simplicity, we restrict attention here to the situation with wave vector orthogonal to $\vec{\beta}$, so $\vec{p} \cdot \vec{\beta}=0$. In this case, the ordinary mode $A_{o}^{\mu}$ can be chosen to satisfy $A_{o}^{0}=0$ with $\vec{A}_{o}$ parallel to $\vec{p} \times \vec{\beta}$, while the extraordinary mode must satisfy $A_{e}^{0}=0$ and has $\vec{A}_{e}$ aligned along $\vec{\beta}$. These two modes propagate with different velocities. For example, their group velocities $\vec{v}_{g} \equiv \vec{\nabla}_{p} p^{0}$ are 


$$
\vec{v}_{g, o}=\hat{p}, \quad \vec{v}_{g, e}=\frac{1}{\sqrt{1+\vec{\beta}^{2}}} \hat{p} .
$$

For each mode, the group and phase velocities are equal.

One consequence of the difference between the two modes is birefringence. For example, a plane-polarized monochromatic wave of frequency $p^{0}$ that is initially a general combination of the two modes eventually becomes elliptically polarized. For the electric field, we find

$$
\begin{aligned}
\vec{E}(t, \vec{x})= & -p^{0}\left(c_{o} \hat{A}_{o} \sin \left[p^{0}(r-t)\right]\right. \\
& \left.+c_{e} \hat{A}_{e} \sin \left[p^{0}\left(\sqrt{1+\vec{\beta}^{2}} r-t\right)\right]\right),
\end{aligned}
$$

where $r=|\vec{x}|, \hat{A}_{o}$ is parallel to $\vec{p} \times \vec{\beta}$ and $\hat{A}_{e}$ is parallel to $\vec{\beta}$ as before, and the weights $c_{o}$ and $c_{e}$ are determined by the initial polarization condition. This shows that the presence of $\vec{\beta}$ causes the wave to become elliptically polarized after it has traveled a distance

$$
r \simeq \frac{\pi}{2\left(\sqrt{1+\vec{\beta}^{2}}-1\right) p^{0}}
$$

The magnetic field exhibits similar behavior.

The explicit expressions for the electric and magnetic fields can be used to derive the energy density $\Theta_{e}^{00}$ and the Poynting vector $\Theta_{e}^{j 0}$ for the extraordinary mode. We find

$$
\Theta_{e}^{00}=\vec{p}^{2} c_{e}^{2} \sin ^{2} p_{\mu} x^{\mu}, \quad \Theta_{e}^{j 0}=p^{0} p^{j} c_{e}^{2} \sin ^{2} p_{\mu} x^{\mu}
$$

This shows that in the present case the velocity of energy transport $v_{e}^{j} \equiv \Theta_{e}^{j 0} / \Theta_{e}^{00}$ is identical to the group and phase velocities, Eq. (54). Some comments about the various velocities in the general case are made in the next subsection.

\section{Analogy to macroscopic media}

In Sec. IV B, an approximate analogy was noted between the equations of motion for the Lorentz-breaking extension of electrodynamics and those for electrodynamics in moving media. In this section, we introduce some useful quantitative analogies between the extended electrodynamics and the electrodynamics of macroscopic media. These can be used to gain further insight about the nature of the extended electrodynamics with Lorentz breaking.

Consider first the situation in position space, where the relevant equations are Eqs. (38) and (39). We have already noted that Eqs. (39) take the same form as in conventional electrodynamics. The idea is to define new quantities $\vec{D}$ and $\vec{H}$ such that the forms of Eqs. (38) become identical to those of the Maxwell equations in material media. It turns out that it suffices to introduce an effective displacement current $\vec{D}$ and an effective magnetic field $\vec{H}$ having linear dependence on the electric field $\vec{E}$, the magnetic induction $\vec{B}$, the vector potential $\vec{A}$, and the scalar potential $A^{0}$.

We find that the definitions

$$
\begin{aligned}
D^{j}= & E^{j}-2\left(k_{F}\right)^{0 j 0 k} E^{k}+\left(k_{F}\right)^{0 j k l} \epsilon^{k l m} B^{m}+2 \epsilon^{j k l}\left(k_{A F}\right)^{k} A^{l}, \\
H^{j}= & B^{j}+\frac{1}{2}\left(k_{F}\right)^{p q r s} \epsilon^{p q j} \epsilon^{r s k} B^{k}-\left(k_{F}\right)^{0 m k l} \boldsymbol{\epsilon}^{j k l} E^{m} \\
& -2\left(k_{A F}\right)^{0} A^{j}+2\left(k_{A F}\right)^{j} A^{0}
\end{aligned}
$$

reproduce the usual Maxwell equations in material media. The analogy can therefore be used to gain insight into those properties of the extended electrodynamics that are directly associated with the equations of motion. However, caution is required in applying other concepts of conventional electrodynamics. For example, it turns out that if $k_{A F} \neq 0$ then the conventional expressions for the energy density and Poynting vector in terms of $\vec{D}$ and $\vec{H}$ fail to reproduce completely the true energy density $\Theta^{00}$ and Poynting vector $\Theta^{j 0}$ in the extended theory. If $k_{A F}=0$, in contrast, the correct expressions are indeed reproduced by the analogy.

The above analogy is useful for general discussions of the properties of the extended electrodynamics. However, it becomes somewhat cumbersome for certain considerations involving radiation. We have developed a second analogy that is of more direct use when the fields are converted to momentum space through Eq. (45). It turns out that the equations of motion can then be correctly reproduced by defining an effective displacement current $\vec{D}(p)$ through

$$
D^{j}=\epsilon^{j k} E^{k}
$$

where $\epsilon^{j k}$ is a Hermitian effective permittivity given by

$$
\boldsymbol{\epsilon}^{j k} \equiv \delta^{j k}+\frac{2}{\left(p^{0}\right)^{2}}\left(k_{F}\right)^{j \beta \gamma k} p_{\beta} p_{\gamma}+\frac{2 i}{\left(p^{0}\right)^{2}}\left(k_{A F}\right)_{\beta} \epsilon^{j \beta \gamma k} p_{\gamma} .
$$

In particular, it is unnecessary to introduce an effective magnetic field $\vec{H}$ distinct from $\vec{B}$. This second analogy is therefore different from the first. Note that again the correct energy density and Poynting vectors cannot be obtained directly by substitution into the conventional formulas. Nonetheless, the analogy is valuable because it permits insight into the effects of Lorentz violation on radiation. Note also that the effective permittivity (60) depends on the frequency $p^{0}$ and wave vector $\vec{p}$, which implies a nonlocal connection between $\vec{D}(x)$ and $\vec{E}(x)$.

The extended Maxwell equations for this analogy directly yield

$$
\vec{p} \cdot \vec{B}=\vec{p} \cdot \vec{D}=\vec{E} \cdot \vec{B}=\vec{D} \cdot \vec{B}=0
$$

The natural right-handed triad of orthonormal vectors describing the vibration of the electromagnetic field is therefore $(\hat{p}, \hat{D}, \hat{B})$. Unlike the case of conventional vacuum radiation, the electric-field vector $\vec{E}$ here is orthogonal only to $\hat{B}$ and so lies off-axis in the $\hat{p}-\hat{D}$ plane. In this analogy, the energy density is typically transported neither in the direction $\hat{p}$ nor in the direction $\hat{E} \times \hat{B}$.

It is useful to introduce a generalized refractive index $n(p)$ by $n(p) \equiv|\vec{p}| / p^{0}$. Its inverse is the magnitude of the 
phase velocity of the mode (45). Using the extended Maxwell equations, we can then deduce the result

$$
\vec{D}=n^{2}[\vec{E}-(\hat{p} \cdot \vec{E}) \hat{p}] \equiv n^{2} \vec{E}_{\perp},
$$

which determines the effective displacement current directly in terms of the electric field and the momentum. Eliminating $\vec{D}$ via Eq. (59) produces a set of three linear equations of the form (47) for $\vec{E}$, with the matrix $M$ now given by

$$
M^{j k} \equiv n^{2} \delta^{j k}-\frac{p^{j} p^{k}}{\left(p^{0}\right)^{2}}-\epsilon^{j k} .
$$

A nontrivial solution exists if $\operatorname{det}\left(M^{j k}\right)=0$. We have explicitly verified that this condition is equivalent to the condition of the vanishing of the determinant of the matrix $M^{j k}$ appearing in Eq. (43) in Sec. IV B.

In conventional crystal optics, the permittivity is often diagonalized: $\epsilon^{j k} \equiv \epsilon^{j} \delta^{j k}$ (no sum), where the eigenvalues $\epsilon^{j}$ are real. This means that the coordinate axes are identified with the principle dielectric axes, which typically represents a different coordinate system than the special one with $\vec{p}$ $=(0,0, p)$ used in Sec. IV B. A diagonalization of this type is also possible in the present analogy because the effective permittivity is Hermitian. Substitution into Eq. (63) and expansion of the determinant then produces an expression with the form of the Fresnel equation of crystal optics. The sixthorder terms in the determinant cancel, ultimately by virtue of the existence of only two independent degrees of freedom in $\vec{E}$. Solving the determinant condition provides the dispersion relations for the independent degrees of freedom. If $\hat{p}$ is given, the condition specifies $p^{0}$ as a function of $|\vec{p}|$. If $p^{0}$ is given, the condition specifies $|\vec{p}|$ as a function of $\hat{p}$.

The special choice of coordinate system in Sec. IV B permits a direct demonstration with this analogy that for a given momentum $\vec{p}$ the solutions for the effective displacement current $\vec{D}$ are linearly polarized. First, substitute for $E^{j}$ $=\left(\epsilon^{-1}\right)^{j k} D^{k}$ in Eq. (62). Using observer rotation invariance to select a frame in which $\vec{p} \equiv(0,0, p)$ then yields the two simultaneous equations

$$
\left[\delta^{a b}-n^{2}\left(\epsilon^{-1}\right)^{a b}\right] D^{b}=0,
$$

where $a, b=1,2$. The vanishing of the determinant of the expression in parentheses generates the analog of the Fresnel equation in this special coordinate system. It can be seen directly from Eq. (64) that for fixed $\vec{p}$ the $\vec{D}$ vectors for each of the two values of $n$ must lie along the principal axes of symmetry of the two-dimensional matrix $\left(\epsilon^{-1}\right)^{a b}$. These two $\vec{D}$ vectors are perpendicular, so an electromagnetic wave corresponding to either one is necessarily linearly polarized.

Many other concepts of crystal optics can be applied in the context of this analogy, including the wave-vector and ray surfaces and the Fresnel and other ellipsoids. The presence of a Lorentz violation means that the vacuum as experienced by an electromagnetic wave behaves like a special kind of crystal. Our results show that the effective medium is optically anisotropic and gyrotropic and exhibits spatial dis- persion of the axes. The earliest mention of effects of this type appeared in an 1878 paper of Lorentz [23], and they are now well established in a variety of physical systems [24]. Thus, the momentum dependence of the effective permittivity corresponds to spatial dispersion of the axes. A nonzero $k_{A F}$ produces a contribution to the effective permittivity analogous to the effects of natural optical activity in a gyrotropic crystal, while a nonzero $k_{F}$ produces effects analogous to spatial dispersion in an optically inactive and anisotropic crystal. Partly on the basis of the hermiticity of the effective permittivity, we also anticipate that in the presence of Lorentz violation the vacuum behaves like a transparent (nonabsorptive) medium, although a complete and elegant demonstration of this remains an open issue.

The above analyses partially simplify if certain components of $k_{F}$ and $k_{A F}$ vanish. Suppose for definiteness that $k_{A F}$ indeed vanishes and that the only nonzero components of $k_{F}$ are $\left(k_{F}\right)^{0 j 0 k}$ and components related to these by the symmetries of $k_{F}$. This makes the effective permittivity real and independent of $p^{\mu}: \epsilon^{j k}=\delta^{j k}-2\left(k_{F}\right)^{0 j 0 k}$. It is then possible, for example, to solve explicitly for the behavior if $\vec{E}$ is specified, which provides yet another approach to the physics. In this case, $\vec{E}_{\perp}$ is the component of $\vec{E}$ perpendicular to $\hat{p}$ in the $\hat{E}-\hat{p}$ plane. It follows that $\vec{E}_{\perp}=(\vec{E} \cdot \hat{D}) \hat{D}$, from which one can derive

$$
\hat{p}=\frac{(\vec{D})^{2} \vec{E}-(\vec{E} \cdot \vec{D}) \vec{D}}{\sqrt{\left[(\vec{E})^{2}(\vec{D})^{2}-(\vec{E} \cdot \vec{D})^{2}\right](\vec{D})^{2}}}
$$

provided $\vec{D}$ and $\vec{E}$ are not parallel. The phase velocity is given by

$$
v_{p}=1 / n=\sqrt{(\vec{E} \cdot \vec{D}) /(\vec{D})^{2}} .
$$

For instance, if $\vec{E}=(E, 0,0)$ then to lowest order in $k_{F}$ we find $\vec{D} \approx|\vec{E}|\left[1-2\left(k_{F}\right)^{0101},-2\left(k_{F}\right)^{0201},-2\left(k_{F}\right)^{0301}\right]$ and $v_{p}$ $\approx 1+\left(k_{F}\right)^{0101}$, which in the appropriate limit agrees with the result for the extraordinary mode of the example at the end of Sec. IV B. Even in this relatively simple case, Eq. (65) shows that the vector $\vec{p}$ can have a complicated structure with components in all three directions.

The above analysis uses the notion of the phase velocity $v_{p}$. However, even in conventional electrodynamics there are numerous possible definitions of the velocity $v$ of an electromagnetic wave, including among others the group velocity, the velocity of energy-momentum transport, and the signal velocity [25]. The Lorentz violation adds further complications to this situation. In the remainder of this subsection, we comment on some aspects of this issue.

An important feature is that the fundamental physical constant $c=1$ relating the space and time components of the metric is unaffected by the Lorentz violation. The underlying spacetime structure of the theory is the usual one because the apparent Lorentz breaking at the level of the standard model is merely a reflection of the presence of nonzero tensor expectation values in a fundamental theory with Lorentzcovariant dynamics. Indeed, $c$ is an invariant under both ob- 
server and particle boosts. However, the physical velocity of an electromagnetic wave can be affected. The situation is analogous to that of a fermion mass parameter $m$ in the Lagrangian for the standard-model extension: although $m$ remains unchanged, the physical rest mass of a particle can be affected [2].

As in conventional electrodynamics, the various definitions of physical velocity are inequivalent in general. Any choice for the physical velocity $v$ typically differs from $c$, although $v \approx c$ since $k_{F}$ and $k_{A F}$ are small. The analyses above indicate that, for any given definition, the magnitude and direction of the velocity of an electromagnetic wave can vary with the wave-vector orientation and the polarization. Incidentally, conventional crystal-optics experiments suggest that there is no general condition requiring the velocity of one type of polarization to exceed the other. For example, the indices of refraction $n_{o}$ and $n_{e}$ for the ordinary and extraordinary rays, respectively, of the sodium $D$ line are measured to be $n_{o}=1.658>n_{e}=1.486$ in calcspar but are $n_{o}$ $=1.544<n_{e}=1.553$ in quartz [26].

For the special case involving Eq. (66), $v_{p}$ may exceed $c$ if the sign of $k_{F}$ is appropriate. In conventional electrodynamics, a phase velocity exceeding $c$ is known to occur in numerous physical situations, for example, for transverse electric (TE) and transverse magnetic (TM) modes in wave guides. Indeed, both the phase and group velocities can simultaneously exceed $c$ in certain refractive materials. For the present theory, it is an open issue to demonstrate that a phase velocity exceeding $c$ is compatible with microcausality. It is possible in principle that only certain sign choices for the components of $k_{F}$ lead to physically acceptable microcausal theories. If this occurs, it would be analogous to the usual requirement of a particular sign for the mass-squared term in a (stable) scalar field theory. In any event, a satisfactory proof of microcausality would involve a complete treatment at the level of quantum field theory and lies beyond the scope of the present work.

Another issue involving the physical velocity $v$ of an electromagnetic wave is its behavior under Lorentz transformations. Since $c$ is invariant under an observer Lorentz transformation whereas $k_{A F}$ and $k_{F}$ change, $v$ is expected to transform along with the frequency and wavelength. This is unlike the conventional case and is a consequence of the presence of the background expectation values. In contrast, a particle Lorentz transformation, which for a fixed polarization mode involves remaining in the specified observer frame but changing $\vec{p}$, has no effect on $c, k_{A F}$, or $k_{F}$. Note that if $\vec{p}$ is changed while the polarization is fixed, the above analyses show that the frequency $p^{0}$ also changes in this case. One might instead countenance another kind of boost in which $\vec{p}$ is changed but $p^{0}$ is unaffected, in which case the polarization must also change.

\section{Constraints from birefringence}

The existence of distinct dispersion relations for the independent polarizations means that birefringence is a major feature of the behavior of an electromagnetic wave in vacuum in the presence of Lorentz violation. In this subsec- tion, we investigate some of the theoretical and experimental implications of a birefringent vacuum.

For definiteness, consider a monochromatic electromagnetic wave of frequency $p^{0}$. The electric field $\vec{E}(t, \vec{x})$ of this wave is formed in general from two independent polarization components:

$$
\begin{aligned}
\vec{E}(t, \vec{x})= & {\left[\vec{E}_{1}\left(\vec{p}_{1}\right) \exp \left(i \vec{p}_{1} \cdot \vec{x}\right)\right.} \\
& \left.+\vec{E}_{2}\left(\vec{p}_{2}\right) \exp \left(i \vec{p}_{2} \cdot \vec{x}\right)\right] \exp \left(-i p^{0} t\right) .
\end{aligned}
$$

The wave vectors $\vec{p}_{1}$ and $\vec{p}_{2}$ must satisfy the appropriate dispersion relations for the specified frequency $p^{0}$. Note that the direction of wave propagation must also be specified to fix completely the solution. One possible determining method could be to require that both component waves propagate their energy density in a given direction.

Since the Lorentz violation is small, we expect $\vec{p}_{2}=\vec{p}_{1}$ $+\delta \vec{p}$, where $\delta \vec{p}$ is small relative to $\vec{p}_{1} \approx \vec{p}_{2}$. Substitution gives

$\vec{E}(t, \vec{x}) \approx\left[\vec{E}_{1}\left(\vec{p}_{1}\right)+\vec{E}_{2}\left(\vec{p}_{2}\right) \exp (i \delta \vec{p} \cdot \vec{x})\right] \exp \left(-i p^{0} t+i \vec{p}_{1} \cdot \vec{x}\right)$.

This equation shows that the birefringence length scale is $|\delta \vec{p}|^{-1}$, which is large when $|\delta \vec{p}|$ is small. Since $\delta \vec{p}$ has dimensions of mass and since it vanishes in the absence of Lorentz violation, its dominant terms are expected to be controlled by $k_{A F}$, by a product of components of $\vec{p}$ and $k_{F}$, or by some combination of the two. This is in agreement with the discussion of the dispersion relations in previous subsections. Note that the associated phase shift $\Delta \phi \equiv \delta \vec{p} \cdot \vec{x}$ cannot correctly be regarded as a phase difference between two circular-polarization modes because typically no such modes exist as solutions of the dispersion relations.

In the remainder of this subsection, we consider possible bounds on $k_{F}$ and $k_{A F}$ from some terrestrial, solar system, astrophysical, and cosmological experiments.

First, we summarize the case of nonzero $k_{A F}$ but zero $k_{F}$. A term of the form (25) appears to have been introduced independently on several occasions, including among others in Ref. [27] and the review [15] mentioned earlier, although the observation that it is $C P T$ violating appears to have been overlooked prior to our earlier work [2]. Given the theoretical difficulties arising from negative contributions to the energy as described in Sec. IV B, it seems possible that this term would need to be absent in nature even if Lorentz symmetry is violated. However, this too is a suggestion that could be the subject of tests.

In a pioneering work [22], Carroll, Field, and Jackiw investigated some properties of the term (25) and used geomagnetic constraints and limits on cosmological birefringence of radio waves to bound certain forms of the coupling coefficient $k_{A F}$. Their treatment of geomagnetic constraints is based on known bounds on the photon mass [28], and it constrains a term of the form $(25)$ with $\left(k_{A F}\right)^{\mu}=(k, \overrightarrow{0})$ to $|k| \lesssim 6 \times 10^{-26} \mathrm{GeV}$. In contrast, the constraints they obtain from cosmological birefringence are considerably sharper, primarily because the distance scales are greater. Their in- 
vestigation seeks a redshift dependence in the established correlation [29] between the intrinsic position angles and the polarization angles of a set of radio galaxies and quasars at distances comparable to the Hubble length. It constrains a particular combination of the coefficients for a timelike $\left(k_{A F}\right)^{\mu}$ to $\leqslant 2 \times 10^{-42} \mathrm{GeV}$. A more recent analysis [30] claims a nonzero observed effect with a spacelike $\left(k_{A F}\right)^{\mu}$ at a scale of approximately $10^{-41} \mathrm{GeV}$. This has been disputed by other authors [31].

We next consider the case with $k_{F} \neq 0$ but $k_{A F}=0$. In its general form, this possibility appears to have been largely disregarded in the literature. However, the rotationally invariant term of the form $\frac{1}{2} \alpha\left(\vec{B}^{2}+\vec{E}^{2}\right)$ in the extended-QED Lagrangian (32) has been considered by several authors, usually in the rescaled form involving only $\vec{B}^{2}$. In particular, this term has a counterpart in the TH $\epsilon \mu$ formalism [32]. This formalism is a phenomenological parametrization for the motion and electromagnetic interactions of charged pointlike test particles in an external spherically symmetric and static gravitational field. It has been extensively used for quantitative tests of the foundations of gravity, including local Lorentz invariance. In this context, clock-comparison experiments have constrained the analog of the parameter $\alpha$ to better than about one part in $10^{21}$ [18]. An improvement over this bound of about an order of magnitude may be possible based on the existence and properties of high-energy cosmic rays [33].

In the general case with nonzero $k_{F}$ and violation of rotational invariance, the sharpest bounds are likely to emerge once again from observational constraints on cosmological birefringence. However, the discussion following Eq. (68) shows there is a significant difference in the $k_{F}$ case: the phase shift $\Delta \phi$ here depends on a product of components of $k_{F}$ and $\vec{p}$, whereas in the $k_{A F}$ case $\Delta \phi$ depends only on $k_{A F}$. This behavior can be seen explicitly, for example, in the special analytical solution presented at the end of Sec. IV B.

The linear dependence of $\Delta \phi$ on momentum or wave number implies an inverse dependence on wavelength. The rotation measures and intrinsic position angles of radio sources [29] are obtained by a fitting procedure that assumes a quadratic dependence on wavelength (proportional to the rotation measure and attributed to Faraday rotation) with a wavelength-independent zero offset (the intrinsic position angle). This procedure is suitable for obtaining constraints on $k_{A F}$, which would generate an extra wavelength-independent effect, but may be inadequate to place a reliable bound on $k_{F}$ or to detect the associated wavelength-dependent effects. It therefore appears somewhat involved to obtain an accurate estimate of the constraints on $k_{F}$ from cosmological birefringence.

Although a complete treatment lies outside our present scope, a crude estimate of an attainable bound on $k_{F}$ can readily be found. It is plausible to suppose that the results of a careful analysis would provide a limit on a product of certain components of $k_{F}$ and $\vec{p}$ comparable to that of order $10^{-42} \mathrm{GeV}$ obtained for $k_{A F}$ in Ref. [22]. The radio sources typically involve wavelengths of order $10 \mathrm{~cm}$, which corresponds to an inverse wavelength of about $10^{-15} \mathrm{GeV}$. This suggests that an upper bound of approximately $10^{-27}$ could be placed on at least some of the (dimensionless) coefficients $k_{F}$. The tightness of this constraint and the apparent feasibility of the analysis suggests this investigation would be worthwhile to pursue. Ideally, a complete study would obtain combined bounds on both of the coupling coefficients $k_{F}$ and $k_{A F}$.

An interesting implication of the (inverse) wavelength dependence of the birefringence is that shorter wavelengths are more sensitive to the effects. Although it may be infeasible in practice, a measurement of cosmological birefringence comparable to the above but obtained with, say, optical sources would be much more sensitive to possible effects from $k_{F}$. Optical wavelengths are a factor of about $10^{-6}$ of radio wavelengths, which would correspond to a millionfold improvement in sensitivity to $k_{F}$.

Other bounds on Lorentz violation could be deduced. In the next section, we show that one-loop radiative corrections induce a dependence of $k_{F}$ on the coefficients $c_{\mu \nu}$ in Eq. (22) for the extended QED. This suggests that if a tight bound were obtained on $k_{F}$ as above, an indirect constraint might also be inferred on $c_{\mu \nu}$. The latter constraint would be weaker by a factor of the fine-structure constant, but the limits deduced would nonetheless probably be comparable to the best ones attainable in other tests of Lorentz symmetry.

If a nonzero effect is detected in the future, it might be of some theoretical interest to investigate the possibility of a correlation between the particular coupling coefficients involved and the motion of the Earth relative to the cosmic microwave background radiation. The point is that the apparent Lorentz violation induces boost (and orientation) dependence in experiments [6]. Although the standard-model extension strictly has no preferred frame, the coupling coefficients must take a canonical form in some observer frame [2]. If the latter is at rest with respect to the cosmic microwave background radiation, a small deviation from the canonical form might arise from the Earth's motion. Although the Earth's speed in this frame is about $10^{-3} c$, the sensitivity of the birefringence measurements might nonetheless be sufficient to detect its effects.

\section{RADIATIVE CORRECTIONS}

We next examine some radiative corrections to the purephoton sector. In Sec. V A, $C P T$-odd terms are investigated. Of particular interest is whether the tree-level vanishing of the coefficient $k_{A F}$ in Eq. (25) for the QED extension, which would eliminate negative contributions to the energy, is reasonable in the light of quantum effects. The point is that the latter might in principle induce a nonzero coefficient through radiative corrections from another sector of the theory. Other quantum corrections that might generate an instability through the linear term $-\left(k_{A}\right)_{\mu} A^{\mu}$ are considered at the end of this subsection. In Sec. V B, we study quantum corrections in the $C P T$-even sector, involving the coefficient $k_{F}$.

The analysis in this section is based on the quantization discussed in Ref. [2]. It is largely at the one-loop level and for leading-order Lorentz-violating effects, and it is primarily limited to issues involving radiative corrections to the 
pure-photon sector. A few results are also presented for higher loops and effects in other parts of the standard-model extension.

An interesting issue indirectly related to some calculations in Sec. V A is whether the anomaly cancellations occurring in the conventional standard model still hold for its extension presented in Sec. II. Three known types of chiral gauge anomaly are relevant [34]. It lies beyond our present scope to provide a complete analysis of all these in the presence of the Lorentz-violating terms, and it is certainly conceivable that the latter would modify the standard derivations. We do expect, however, that the usual cancellations of the (Abelian, singlet, and non-Abelian) triangular gauge anomalies and the nonperturbative global SU(2) anomaly indeed remain valid. The point is that the standard-model extension has the same multiplets and the same gauge structure as the conventional case, so the group-theoretic underpinnings of the usual analyses are unaffected. The situation for the third type of anomaly, which is the mixed gaugegravitational chiral anomaly associated in part with local Lorentz transformations, is less clear. The presence of Lorentz violations might appear to suggest a potentially nonzero contribution to this anomaly. However, a careful analysis is needed because observer Lorentz invariance is in fact maintained in the standard-model extension.

\section{A. $C P T$-odd terms}

As discussed above, the possible difficulties with negative contributions to the energy and the tight experimental constraints suggest that the coefficient $k_{A F}$ vanishes. If it is set to zero at tree level, the issue arises as to whether it acquires radiative corrections from quantum loop corrections. If so, there could be both theoretical and experimental arguments suggesting associated constraints on certain other coefficients in the standard-model extension. The issue of the vanishing of radiative corrections to $k_{A F}$ therefore has the potential to provide a nontrivial consistency check on the theory. In the present subsection we investigate this, assuming that $k_{A F}$ is zero at the tree level and beginning with one-loop effects at leading order in the Lorentz-breaking terms. Remarkably, as we show next, the structure of the standardmodel extension is such as to preserve a vanishing coefficient $k_{A F}$ at this level.

A radiative contribution to $k_{A F}$ would represent a correction to the photon propagator. In the standard-model extension, the Feynman rules for leading-order effects from Lorentz-violating terms take the form of insertions on propagators or at vertices already existing in the conventional theory [2]. Also, the photon interacts with charged particles as usual, so the only possible diagrams modifying the photon propagator at the one-loop level are those of the standard one-loop vacuum polarization but with an insertion either on an internal charged-particle line or at one of the vertices.

The apparently daunting task of examining every possible insertion implied by the extra terms in the standard-model extension can be simplified by taking advantage of the discrete operations $C, P$, and $T$. A radiative term purporting to contribute to the coefficient $k_{A F}$ must have appropriate trans- formation properties under these discrete symmetries. In particular, it must be $C$ even and $P T$ (and $C P T$ ) odd, although either of the two possible combinations of $P$ and $T$ could occur. At the level of the QED extension with electrons and positrons, the only term of this type is the one with coupling coefficient $b_{\mu}$ in Eq. (28). This is true even if the discarded linear term $-\left(k_{A}\right)_{\kappa} A^{\kappa}$ in the Lagrangian were present, which is $C$ and $C P T$ odd. At any loop order, contributions must therefore involve an odd number of line insertions arising from the term with coefficient $b_{\mu}$. At the one-loop level in the full standard-model extension, similar terms involving the other lepton and quark fields would also contribute to appropriate internal lines. However, only one additional distinct type of one-loop contribution appears, involving a vertex correction proportional to the coefficient $\left(k_{2}\right)_{\mu}$ in Eq. (17) in a diagram with a $W^{+}-W^{-}$loop. The demonstration that no net contributions to $k_{A F}$ arise at one loop therefore involves consideration of only terms involving the $b_{\mu}$-type and the $\left(k_{2}\right)_{\mu}$ coefficients.

Excluding the external photon legs, any contributions to the vacuum polarization must have dimensions of mass squared. The leading-order contribution to $k_{A F}$ must involve both a momentum factor from the necessary derivative on an external leg and one power of either $b_{\mu}$ or $\left(k_{2}\right)_{\mu}$. Since these factors already give the correct dimensionality, any others must appear in dimensionless combinations of the photon momentum $p^{\mu}$ and the mass $m$ of the particle in the loop. This is confirmed by the explicit calculation below.

We first consider corrections to the one-loop vacuum polarization involving $b_{\mu}$. Each such two-point diagram has the usual form except for an insertion of the factor $-i b_{\mu} \gamma_{5} \gamma^{\mu}$ on one internal fermion line. From the perspective of the fundamental theory, a one-loop two-point diagram with a fermion-line insertion is closely related to a one-loop three-point diagram containing the same two photon legs together with a third leg involving a coupling to an axial vector. A fermion-line insertion in the two-point diagram can then be viewed as a limit of this three-point diagram in which there is zero momentum transfer to the axial-vector leg and the axial vector is replaced with a vacuum expectation value.

This line of reasoning is interesting because a one-loop three-point diagram with an axial-vector and two photon couplings is directly related to a triangular gauge anomaly. If the axial vector is a gauge field in the underlying theory, such anomalies must cancel for the theory to be renormalizable. One might therefore conjecture that the cancellation of these anomalies could also imply cancellation of the limiting two-point diagrams in the standard-model extension. If true, this provides another link relating consistency of the standard-model extension to the spontaneous nature of the Lorentz violation in the underlying theory. Next, we develop a line of reasoning that provides insight into this question.

Independently of the issue of corrections to the photon propagator, the requirement that the triangular anomalies cancel in the underlying theory implies a constraint on coefficients of the type $b_{\mu}$ that is of interest in its own right. It turns out that this constraint is relevant to the photon propagator, so we begin by deriving it. 
Consider first the origin in the underlying theory of the axial-vector coupling in the triangle diagram. Prior to spontaneous symmetry breaking, the fundamental Lagrangian may contain several terms of the general form $g_{\psi}^{a}\left(T^{a}\right)_{\mu \nu \ldots \rho} \bar{\psi}\left(\Gamma^{a}\right)^{\mu \nu \ldots \rho} \psi$ for each fermion species $\psi$, where $T^{a}$ is a tensor field, $\Gamma^{a}$ is a gamma-matrix structure, $g_{\psi}^{a}$ is the associated coupling constant, and $a$ is a label ranging over the set of tensor fields that couple to the species $\psi$. Note that the only acceptable line insertions in the two-point one-loop diagram are flavor diagonal, so in the present context contributions are possible only from the diagonal components of Eqs. (10) and (12). We therefore disregard possible cross couplings between fermion species in this derivation.

Each of these Lagrangian terms can be decomposed in terms of the usual 16 basis $\gamma$ matrices in four dimensions. Collecting terms produces for each fermion species a Lagrangian separated into five parts, one for each of the five types of fermion bilinear: scalar, pseudoscalar, vector, axialvector, and tensor. The particular components of the fields $T^{a}$ that multiply the axial-vector bilinear can be regarded as a set of effective axial vectors $A_{5 \mu}^{a}$ with associated coupling constants $g_{\psi}^{a}$. These axial vectors are the fields relevant for the one-loop three-point diagrams of interest. When the axial vectors $A_{5 \mu}^{a}$ acquire vacuum expectation values $\left\langle A_{5 \mu}^{a}\right\rangle$, their net contribution generates the coupling coefficient $b_{\mu}^{\psi}$ $\equiv \Sigma_{a} g_{\psi}^{a}\left\langle A_{5 \mu}^{a}\right\rangle$ at the level of the standard-model extension for this species of fermion.

The triangle diagram for one axial vector $A_{5 \mu}^{a}$ and two photons $A_{\mu}$ has an anomaly proportional to the product of $g_{\psi}^{a}$ and $q_{\psi}^{2}$, where the latter is the charge of the fermion $\psi$. When summed over all fermion species, the anomalycancellation condition is therefore

$$
\sum_{\psi} q_{\psi}^{2} g_{\psi}^{a}=0
$$

for each $a$. Multiplying this equation by $\left\langle A_{5 \mu}^{a}\right\rangle$ and summing over $a$ yields the constraint

$$
\sum_{\psi} q_{\psi}^{2} b_{\mu}^{\psi}=0
$$

on coupling coefficients of the $b_{\mu}$ type. Note that, at the level of the standard-model extension, the sum over all fermion species would include the leptons and the quarks. Also, in contrast to the usual anomaly-cancellation mechanism which produces a single condition, Eq. (70) is a set of four constraints. This is a direct consequence of spontaneous Lorentz breaking, in which for each $a$ the vacuum expectation value $\left\langle A_{5 \mu}^{a}\right\rangle$ involves four numbers.

Next, we present the results of an explicit calculation of the $b_{\mu}$-linear one-loop corrections to the photon propagator involving a fermion of mass $m$ and charge $q$. There are two diagrams to consider, since a factor $-i b_{\mu} \gamma_{5} \gamma^{\mu}$ can be inserted on either of the two internal lines. Using an argument similar to the standard one proving the Furry theorem, the two diagrams can be shown to give identical contributions to the amplitude. Omitting the external photon legs, the correction to the two-point amplitude for a photon of fourmomentum $p^{\mu}$ then becomes

$$
\begin{aligned}
\bar{\omega}^{\mu \nu}(p, m, b)= & -2 i q^{2} b_{\lambda} \int \frac{d^{4} l}{(2 \pi)^{4}} \\
& \times \operatorname{Tr}\left[\gamma^{\mu} S_{F}(l-p) \gamma^{\nu} S_{F}(l) \gamma_{5} \gamma^{\lambda} S_{F}(l)\right],
\end{aligned}
$$

where $l^{\mu}$ is the momentum of the fermion in the loop and $S_{F}(l)=i(l-m+i \epsilon)^{-1}$ is the usual fermion propagator.

As anticipated above, expression (71) is related to one appearing in the calculation of the triangular gauge anomaly. It can directly be verified that

$$
\bar{\omega}^{\mu \nu}(p, m, b) \equiv q^{2} b_{\lambda} T^{\mu \nu \lambda}(-p, p),
$$

where $T^{\mu \nu \lambda}\left(p_{1}, p_{2}\right)$ is the standard amplitude for the triangle diagram with one axial-vector coupling in conventional QED. The full anomaly amplitude $T^{\mu \nu \lambda}\left(p_{1}, p_{2}\right)$ can be regularized in the Pauli-Villars scheme and reduced to a set of integral expressions [35,36]. These can be evaluated in closed form for the present case of interest. For $p^{2}<4 m^{2}$, we find

$$
\begin{aligned}
\bar{\omega}^{\mu \nu}(p, m, b)= & \frac{q^{2} b_{\lambda}}{2 \pi^{2}} p_{\kappa} \epsilon^{\kappa \lambda \mu \nu} \\
& \times\left[1-\frac{4}{\sqrt{\left(p^{2} / m^{2}\right)\left(4-p^{2} / m^{2}\right)}}\right. \\
& \left.\times \tan ^{-1}\left(\sqrt{\frac{p^{2} / m^{2}}{4-p^{2} / m^{2}}}\right)\right] .
\end{aligned}
$$

Note that this expression is gauge invariant,

$$
p_{\mu} \bar{\omega}^{\mu \nu}=p_{\nu} \bar{\omega}^{\mu \nu}=0,
$$

as expected.

At this stage, the issue of radiative corrections to $k_{A F}$ can be addressed. The result (73) is finite. Since no divergence cancellation is necessary, a zero value of $k_{A F}$ at tree level is consistent with a renormalizable theory. Moreover, $\bar{\omega}^{\mu \nu}$ vanishes for the on-shell condition $p^{2}=0$, as is to be expected in a renormalizable theory without a radiatively induced phase transition. Thus, none of the finite radiative corrections have the form needed to modify the coefficient $k_{A F}$, and they are therefore irrelevant to the analysis of cosmic birefringence in Sec. IV D.

The above results might make it seem tempting to conclude that there are no $b_{\mu}$-linear one-loop radiative corrections affecting $k_{A F}$. However, such a conclusion would be premature. The integral $T^{\mu \nu \lambda}(-p, p)$ in Eq. (72) is superficially linearly divergent. As usual, this introduces an ambiguity because a shift in the loop momentum $l^{\mu}$ produces a shift in the value of the integral: $T^{\mu \nu \lambda}(-p, p)$ $\rightarrow T^{\mu \nu \lambda}(-p, p)+\zeta p_{\kappa} \epsilon^{\kappa \lambda \mu \nu}$, where $\zeta$ is a constant. Certain choices of regularization scheme could therefore generate an additional term of the form 


$$
\delta \bar{\omega}^{\mu \nu}(p, b)=\zeta q^{2} b_{\lambda} p_{\kappa} \epsilon^{\kappa \lambda \mu \nu}
$$

to the result (73), which would represent a regularizationdependent radiative correction to $k_{A F}$. Note that this does not occur in the Pauli-Villars scheme because the term (75) is mass independent, so in this case the regularization automatically subtracts it.

Ambiguities of the general form (75) involving combinations of the external momenta arise in the standard triangular-anomaly diagram with a finite momentum transfer from the two photons to the axial vector. In this case, the ambiguity is conventionally fixed by imposing $U(1)$ current conservation. However, in the general context the presence of an ambiguity is independent of the issue of anomalies. Under certain circumstances, anomalies can appear in superficially convergent (non-Abelian pentagon) diagrams that are ambiguity-free [37]. Also, ambiguities originating in loopmomentum shifts for divergent amplitudes other than those associated with anomalies are a standard feature of quantum field theories. For example, the usual vacuum-polarization diagram has an ambiguity. Similarly, results such as the Furry theorem rely on a consistent assignment of loop momenta. Typically, these ambiguities either appear as finite constant modifications to divergent constants or can be eliminated by imposing gauge invariance.

A striking feature of the $\zeta$ ambiguity is that it arises without an associated divergence and is gauge invariant. It therefore cannot be fixed by the usual methods. Thus, gauge invariance ensures the Ward identities are satisfied, so vectorcurrent conservation holds for any $\zeta$. Also, the ambiguity fails to produce an anomaly in the axial-current conservation law because there is zero momentum transfer away from the loop at the axial vertex for any $\zeta$. However, the mass independence of the term (75) implies that the fermion mass circulating in the loop could in principle be arbitrarily large without affecting the value of $\zeta$, which intuitively seems unphysical and would appear to suggest that $\zeta$ must vanish.

In the standard triangular-anomaly diagram, fixing the ambiguity by requiring vector-current conservation places the anomaly in the axial Ward identity. If the axial vector is ungauged, chiral-current conservation is then violated and the anomaly may have physical consequences. An example of this occurs in the decay $\pi \rightarrow 2 \gamma$. If instead the axial vector is a gauge field, then the anomaly destroys renormalizability unless the total anomaly contribution from all fermion species vanishes. A cancellation of this type, which is widely used in model building, implicitly assumes the ambiguity has been fixed in a standard way in all contributing diagrams. This could be regarded as a (reasonable) choice made to obtain a satisfactory theory.

If a similar choice is made for the present case, so that the same regularization scheme is adopted for all the contributing diagrams and therefore the same constant $\zeta$ appears in each, then it can be argued that the anomaly cancellation in the underlying theory causes the ambiguity to disappear. Thus, suppose as above we assume gauged axial vectors in a renormalizable underlying theory, so that the anomalycancellation condition (70) must hold. Then, the net contribution to the photon propagator from the ambiguous terms is given by $\Sigma_{\psi} \zeta q_{\psi}^{2} b_{\lambda}^{\psi} p_{\kappa} \epsilon^{\kappa \lambda \mu \nu}$, which vanishes by Eq. (70). This confirms the conjecture made in the first part of this subsection: the anomaly cancellation implies the absence of $b_{\mu}$-linear one-loop radiative corrections to $k_{A F}$.

Note that this argument presupposes that the axial vectors $A_{5 \mu}^{a}$ are gauged and that a consistent choice of regularization is used. To demonstrate the absence of negative-energy contributions to the theory at this level, it suffices that a natural procedure of this kind exists. If an ambiguity $\zeta$ did remain in the theory, it would seem to suggest that at the quantum level there would be a spectrum of physically allowed theories. The issue of determining the correct one would then become experimental, much as the values of the renormalized couplings and masses are experimentally determined. However, in the present case there are both theoretical and experimental reasons to believe that $\zeta$ vanishes.

We next address some issues arising in higher perturbation orders. Consider first the case of the photon propagator in the extended QED. At any loop order but with only one $C P T$-violating insertion of $b_{\mu}$, all diagrams are superficially divergent and hence can be expected to have ambiguities. In parallel with the previous case, these diagrams can be related to higher-loop three-point triangle diagrams with one axialvector and two photons on the external legs. The AdlerBardeen theorem [38] shows that the anomalies arising from the one-loop triangle diagram are unaffected at higher loops. This implies that the constraint (70) holds at arbitrary loop order. However, it follows as before that the total ambiguity is proportional to this constraint and so vanishes. If this argument holds, then there can be no $b_{\mu}$-linear contributions to $k_{A F}$ at any order in the fine-structure constant.

Diagrams that involve higher-order Lorentz violation may also be of potential theoretical importance. Their transformation properties under discrete symmetries place strong constraints on their possible contributions to $k_{A F}$, as in the lowest-order case. For example, in the extended QED at the quadratic level of Lorentz violation, only a product of the coefficients $b_{\mu}$ and $c_{\mu \nu}$ can appear. At the one-loop level, all higher-order diagrams are related to polygonal diagrams in the underlying theory that couple two photons to a variety of vector, axial-vector, and tensor fields. At least one factor of $b_{\mu}$ is required, so a chiral coupling must be involved and a cancellation mechanism may still apply. The implication of the consistency of the underlying theory for corrections to $k_{A F}$ at all orders in Lorentz violation and including possible higher-loop corrections remains an open issue. We remark, however, that the effects at the cubic levels and above are at most of theoretical interest as they would be well below experimental detection for the levels of Lorentz violation considered in the present work.

At the level of the standard-model extension, a possible lowest-order one-loop correction to the photon propagator could in principle also arise from the coefficient $k_{2}$ when a $W^{+}-W^{-}$pair circulates in the loop. Indeed, there would be a contribution from insertions on the gauge-boson lines and another, related to the first by gauge invariance, involving a modified vertex. However, if the term involving $k_{2}$ were to exist, it would exhibit difficulties with negative contributions to the energy, as does $k_{A F}$. One option is therefore that $k_{2}$ 
vanishes at tree level, which eliminates the possible associated radiative corrections to the term involving $k_{A F}$. An issue then arises concerning possible radiatively induced contributions to $k_{2}$ from the fermion sector. We conjecture that, if all the terms $k_{0}, k_{1}, k_{2}, k_{3}$ vanish at tree level, then no radiative corrections to any of these coefficients arise from the fermion sector. An anomaly-cancellation mechanism would again play a role, although non-Abelian fields would now be involved and so the singlet and non-Abelian anomalies would also be relevant. The situation in the standardmodel extension at higher loops remains open.

Finally, we present a few remarks about the possibility of radiative corrections to a hypothetical linear term of the form $-\left(k_{A}\right)_{\kappa} A^{\kappa}$ in the absence of a photon mass. This term is $C$ and $C P T$ odd. In the extended QED, only terms of the type $a_{\mu}$ have this symmetry. As discussed in Sec. III, a field redefinition can be used to eliminate these (flavor-diagonal) terms, and hence they are unobservable in any experiment. Since the electromagnetic interactions are $C$ even, at lowest order in Lorentz-violating coefficients there cannot be any radiative corrections to $k_{A}$ at any order in QED loops. Any contributions that might arise at higher orders in Lorentz violation would again be related to polygonal diagrams in the underlying theory. It would be of some theoretical interest to investigate the possible contributions to these terms.

\section{B. CPT-even terms}

In contrast to the situation for the $C P T$-odd terms, a nonzero tree-level value for the $C P T$-even term (24) with coefficient $k_{F}$ presents no immediate theoretical difficulty. We have shown in Sec. IV D that it is experimentally feasible to place relatively tight bounds on $k_{F}$ from measurements of cosmological birefringence, although this has not yet been done and the wavelength dependence may result in constraints somewhat weaker than those on $k_{A F}$. Nonetheless, the attainable limits on $k_{F}$ are of interest because they might in principle be sufficiently sharp to be sensitive to effects at a scale comparable to finite radiative corrections from the fermion sector. It is therefore of interest to determine whether the coupling $k_{F}$ must be present for renormalizability and, if so, which fermion-sector coupling coefficients are involved. In this subsection, we investigate this issue in the context of the extended QED.

At the one-loop level and to leading order in Lorentz violation, the possible radiative contributions to the coefficient $k_{F}$ in the term (24) are significantly constrained by the requirements of discrete symmetries. This term is both $C$ and $C P T$ even, and an inspection shows that the only other type of term in the fermion sector with these properties is the term with coefficient $c_{\mu \nu}$ in Eq. (27). It contributes both on the loop through fermion-line insertions with a derivative and at the vertices through the extra gauge coupling.

The form of the $c_{\mu \nu}$-linear correction $\bar{\omega}^{\mu \nu}(p, m, c)$ to the two-point amplitude for a photon of four-momentum $p^{\mu}$ is strongly constrained by its discrete-transformation properties, observer Lorentz covariance, and the requirements (74) of gauge invariance. Thus, invariance under $C P T$ implies $\bar{\omega}^{\mu \nu}(p, m, c)$ is an even function of $p^{\mu}$. Also, by virtue of the definition of the photon propagator as a vacuum expectation value of a time-ordered product, $\bar{\omega}^{\mu \nu}(p, m, c)$ is symmetric under the combination of a sign change $p^{\lambda} \rightarrow-p^{\lambda}$ of the momentum and an interchange $\mu \leftrightarrow \nu$ of the spacetime indices. These conditions imply that the correction to the photon propagator at any order in the fine-structure constant but at linear order in $c_{\mu \nu}$ must take the form

$$
\begin{aligned}
\bar{\omega}^{\mu \nu}(p, m, c)= & i c_{\alpha \beta}\left(A g^{\alpha \beta}\left(p^{2} g^{\mu \nu}-p^{\mu} p^{\nu}\right)\right. \\
& +B\left[\left(p^{2} g^{\alpha \mu} g^{\beta \nu}-g^{\alpha \mu} p^{\beta} p^{\nu}-g^{\alpha \nu} p^{\beta} p^{\mu}\right)\right. \\
& \left.+(\alpha \leftrightarrow \beta)]+C g^{\mu \nu} p^{\alpha} p^{\beta}+\frac{D}{m^{2}} p^{\alpha} p^{\beta} p^{\mu} p^{\nu}\right) .
\end{aligned}
$$

Here, $A, B, C$, and $D$ are (possibly divergent) scalar functions of $p^{2} / m^{2}$ obeying the relationship

$$
C-2 B+\frac{p^{2}}{m^{2}} D=0
$$

to ensure gauge invariance.

Some information about photon propagation under specified circumstances can be deduced from Eq. (76) under the assumption that the scalar functions $A, B, C, D$ have been regularized as needed and divergent contributions have been removed by the renormalization procedure. For example, in the case of cosmological birefringence of interest in Sec. IV D, the photon momentum can be taken as on shell and the Lorentz gauge condition can be applied. In Eq. (76), this corresponds to setting to zero both $p^{2}$ and the momentum factors $p^{\mu}$ and $p^{\nu}$ with specific indices $\mu$ and $\nu$. This leaves only the term $c_{\alpha \beta} C(0) g^{\mu \nu} p^{\alpha} p^{\beta}$. This is precisely of the form needed for radiative corrections to the coefficient $k_{F}$, which can thus be seen to be governed in this gauge by the on-shell value of $C$.

To obtain the explicit result and as a check on the renormalization procedure when Lorentz violations are involved, we have directly performed the one-loop calculation. This also verifies the structure of Eq. (76). The terms in Eq. (27) associated with the coupling coefficient $c_{\mu \nu}$ lead to four new $c_{\mu \nu}$-linear one-loop vacuum-polarization diagrams. The possibility of fermion-line insertions arising from the derivative coupling leads to two diagrams, each with one insertion on one of the two internal fermion lines. The appearance of modified vertices from the extra gauge coupling leads to another two, each with one normal and one modified vertex. These two types of contribution are related by gauge invariance. Indeed, we anticipate this gauge invariance leads to Ward-type identities valid at arbitrary loop order, although an explicit demonstration of this remains an open issue.

The sum of the four additional diagrams generates a oneloop correction to the photon propagator of 


$$
\begin{aligned}
\bar{\omega}^{\mu \nu}(p, m, c)= & i q^{2} c_{\alpha \beta} \int \frac{d^{4} l}{(2 \pi)^{4}}\left\{l^{\beta} \operatorname{Tr}\left[\gamma^{\mu} S_{F}(l-p) \gamma^{\nu} S_{F}(l) \gamma^{\alpha} S_{F}(l)\right]+(l-p)^{\beta} \operatorname{Tr}\left[\gamma^{\mu} S_{F}(l-p) \gamma^{\alpha} S_{F}(l-p) \gamma^{\nu} S_{F}(l)\right]\right. \\
& \left.-i g^{\beta \mu} \operatorname{Tr}\left[\gamma^{\alpha} S_{F}(l-p) \gamma^{\nu} S_{F}(l)\right]-i g^{\beta \nu} \operatorname{Tr}\left[\gamma^{\mu} S_{F}(l-p) \gamma^{\alpha} S_{F}(l)\right]\right\},
\end{aligned}
$$

where the first two terms arise from line insertions and the last two from the modified vertices.

The integral in Eq. (78) is superficially quadratically divergent. It has the standard ambiguity, arising from the possibility of shifting the integration variable, that is (largely) fixed by imposing gauge invariance. The denominators arising from the fermion propagators $S_{F}$ can be combined with the usual Feynman parametrization. All the necessary shifts performed in the resulting integration variables must be the same, so that the contributions from the surface terms remain gauge invariant. To accomplish this, it is convenient to separate $\bar{\omega}^{\mu \nu}$ into two pieces that can be parametrized so as to maintain the equivalence of shifts.

We define $\bar{\omega}^{\mu \nu}=\bar{\omega}_{(1)}^{\mu \nu}+\bar{\omega}_{(2)}^{\mu \nu}$, where

$$
\begin{aligned}
\bar{\omega}_{(1)}^{\mu \nu}= & 4 q^{2} c_{\alpha \beta} \int \frac{d^{4} l}{(2 \pi)^{4}} \frac{1}{\Delta}\left\{g^{\mu \nu}\left[(l-p)^{\alpha} l^{\beta}+(\alpha \leftrightarrow \beta)\right]+\left[g^{\mu \beta}\left[l \cdot(l-p)-m^{2}\right] g^{\nu \alpha}+(\mu \leftrightarrow \nu)\right]\right. \\
& \left.-\left(\left[g^{\mu \alpha}\left[l^{\beta}(l-p)^{\nu}+(\beta \leftrightarrow \nu)\right]+(\mu \leftrightarrow \nu)\right]+(\alpha \leftrightarrow \beta)\right)\right\}
\end{aligned}
$$

and

$$
\begin{aligned}
\bar{\omega}_{(2)}^{\mu \nu}= & 8 q^{2} c_{\alpha \beta} \int \frac{d^{4} l}{(2 \pi)^{4}} \frac{1}{\Delta^{2}}\left\{\left(l^{\alpha} l^{\beta}\left[(l-p)^{2}-m^{2}\right]+(l-p)^{\alpha}(l-p)^{\beta}\left(l^{2}-m^{2}\right)\right)\right. \\
& \left.\times\left(m^{2} g^{\mu \nu}-l \cdot(l-p) g^{\mu \nu}+(l-p)^{\mu} l^{\nu}+l^{\mu}(l-p)^{\nu}\right)\right\} .
\end{aligned}
$$

In these expressions, $\Delta=\left(l^{2}-m^{2}\right)\left[(l-p)^{2}-m^{2}\right]$. The same shift is introduced in all the integrals, thereby preserving gauge invariance, with the substitution in Eq. (79) of

$$
\frac{1}{\Delta}=\int_{0}^{1} d z \frac{1}{\left[k^{2}+z(1-z) p^{2}-m^{2}\right]^{2}}
$$

and the substitution in Eq. (80) of

$$
\frac{1}{\Delta^{2}}=\int_{0}^{1} d z \frac{6 z(1-z)}{\left[k^{2}+z(1-z) p^{2}-m^{2}\right]^{4}},
$$

where $k=l-p z$ is the new integration variable.

The divergences in the resulting integrals can be treated using dimensional regularization in $D=4-\epsilon$ dimensions. Performing various partial integrations, we obtain for $p^{2}$ $<4 m^{2}$ a radiative correction of the form (76) with

$$
\begin{gathered}
A=-B=\omega\left(p^{2} / m^{2}\right), \\
C=-2 \omega\left(p^{2} / m^{2}\right)-\frac{p^{2}}{m^{2}} D, \\
D=2 \frac{\partial}{\partial\left(p^{2} / m^{2}\right)} \omega\left(p^{2} / m^{2}\right) .
\end{gathered}
$$

Here, $\omega\left(p^{2} / m^{2}\right)$ is the standard vacuum-polarization result, given by

$$
\begin{aligned}
\omega\left(p^{2} / m^{2}\right)= & \frac{q^{2}}{4 \pi^{2}}\left[\frac{1}{3}\left(\frac{2}{\epsilon}-\gamma\right)\right. \\
& -2 \int_{0}^{1} d z z(1-z) \ln \left[1-z(1-z)\left(p^{2} / m^{2}\right)\right],
\end{aligned}
$$

where $\gamma$ is the Euler constant. Note that the results (83) satisfy the gauge-invariance condition (77).

The above calculation shows that the scalar function $C$ contains a momentum-independent divergence. As described above, the on-shell value of $C$ determines the coefficient $k_{F}$ in the Lorentz gauge, so the appearance of this divergence shows that a bare coefficient $k_{F}$ must be present in the original theory for renormalizability. The renormalization procedure then removes the infinite and (ambiguous) constant pieces, leaving a physical coefficient $k_{F}$ (to be determined by experiment) and a set of finite radiative corrections governed by the ratio $p^{2} / m^{2}$.

We have seen in Sec. IV D that a nonzero value of the coefficient $k_{F}$ induces cosmological birefringence. The above calculation shows that imposing a zero value of this coefficient at tree level is incompatible with renormalizability. It is therefore reasonable to expect a nonzero physical value of $k_{F}$. Although nonrigorous, a heuristic argument might also be used to provide a relationship between the physical values of $k_{F}$ and $c_{\mu \nu}$ : for consistency of perturbation theory, it is plausible that the physical value of $k_{F}$ should be larger than the expected finite quantum corrections of order $\alpha c_{\mu \nu}$, where $\alpha$ is the fine-structure constant. If $k_{F}$ is 
eventually bounded to about $10^{-27}$ as estimated in Sec. IV D, then this would suggest the components of $c_{\mu \nu}$ might be expected to be smaller than about $10^{-25}$.

As in the $C P T$-odd case, the momentum-dependent radiatively induced corrections in Eq. (83) are irrelevant in the context of cosmological birefringence. However, these radiative corrections do modify the off-shell propagator and might therefore be expected to generate small effects under suitable circumstances. For example, there may be contributions to electromagnetic scattering cross sections, governed by the ratio $\alpha m_{e} / M_{P} \approx 10^{-25}$. Similarly, a small correction to the Coulomb law might appear. These issues lie beyond the scope of the present work.

In addition to the $c_{\mu \nu}$-linear one-loop contribution obtained above, there are also $c_{\mu \nu}$-linear higher-loop corrections in the extended QED. The general structure of the contributions at any loop order is given by Eq. (76). Although the detailed form of the scalar functions $A, B, C$, and $D$ will differ, the physically relevant corrections should also depend on $p^{2} / \mathrm{m}^{2}$, since any terms independent of $p$ are expected to be absorbed by the renormalization procedure. The above conclusions about cosmological birefringence are therefore likely to remain valid. Effects from higher-order Lorentz violation should also arise in the extended QED but are probably of a size that is physically irrelevant. Much of the above discussion should also hold for radiative effects in the full standard-model extension. In this context, note that offdiagonal terms in generation space cannot contribute at leading order.

\section{SUMMARY}

In this paper, we presented a general Lorentz-violating extension of the minimal $\mathrm{SU}(3) \times \mathrm{SU}(2) \times \mathrm{U}(1)$ standard model including both $C P T$-even and $C P T$-odd terms, and we discussed some of its theoretical and experimental properties. The analysis was performed within the context of a framework previously described [2], which is based on spontaneous Lorentz and $C P T$ violation occurring in an underlying theory of nature.

Despite the existence of terms causing a certain type of Lorentz breaking, the resulting theory preserves various desirable features of standard quantum field theories such as gauge invariance, energy-momentum conservation, observer Lorentz invariance, hermiticity, the validity of conventional quantization methods, and power-counting renormalizability. Other important features such as positivity of the energy, microcausality, and the usual anomaly cancellation are also expected. We have demonstrated that the usual breaking of $\mathrm{SU}(2) \times \mathrm{U}(1)$ symmetry to the electromagnetic $\mathrm{U}(1)$ is maintained, although the expectation value of the Higgs is slightly changed and the $Z^{0}$ field acquires a small expectation value. The theory presented here appears at present to be the sole candidate for a consistent extension of the standard model providing a microscopic theory of Lorentz violation.

We have extracted extensions of several of the conventional varieties of QED by considering limiting cases of the standard-model extension. Part of the motivation for investigating extended QED is the existence of high-precision tests of Lorentz and $C P T$ invariance that involve electrodynamics. A summary was provided of some recent studies of possible experimental constraints.

Another major focus of this work is the effect of the Lorentz violations on the photon sector. The general purephoton Lagrangian can be written in a form containing only two additional terms, one $C P T$ odd and one $C P T$ even. This lagrangian and the associated energy-momentum tensor were discussed, and it was found that the $C P T$-even component has positive conserved energy but that in the absence of a photon mass the $C P T$-odd component can generate negative contributions to the energy. Despite this theoretical difficulty, the two terms were retained for the whole analysis so that the implications for the full quantum theory could be examined.

The equations of motion generalizing the Maxwell equations in the presence of Lorentz violation were obtained, and their solution was outlined using both potentials and fields. Some technical complications arise relative to the case of conventional electrodynamics in vacuo, but they can largely be overcome. A key feature is that, although there are still two independent propagating degrees of freedom, in the typical situation the two modes obey different dispersion relations. This implies a variety of interesting effects, including birefringence in the vacuum. We presented a few quantitative analogies with crystal optics and showed that the presence of Lorentz violation means that the vacuum as experienced by an electromagnetic wave behaves like an optically anisotropic and gyrotropic transparent crystal exhibiting spatial dispersion of the axes.

A variety of terrestrial, astrophysical, and cosmological bounds on photon properties are known. Sharp experimental limits on the photon sector of the extended QED can be obtained from the absence of birefringence on cosmological scales. It has been shown in earlier work [22] that the problematic $C P T$-odd term is experimentally limited to scales comparable to the Hubble length.

A significant result of this paper is that most of the components of the $C P T$-even term could also be bounded experimentally from cosmological birefringence with existing techniques. This case is particularly interesting as it has no evident theoretical difficulties and appears to have been overlooked in the previous literature. Also, unlike the $C P T$-odd term, the $C P T$-even contribution exhibits a dependence on wavelength that might provide a useful signature of the effect. We have crudely estimated the attainable bounds, which would be sensitive to suppressed Lorentz violation in the general range considered here.

The paper also contains a series of consistency checks on the theory, primarily at the level of one-loop radiative corrections. We discussed the cancellation of various conventional anomalies in the standard-model extension and considered other anomaly cancellations that might occur in the underlying theory. The latter were used to obtain a constraint on a set of coupling coefficients for Lorentz violation in the standard-model extension.

We have investigated the feasibility of imposing treelevel vanishing of the problematic $C P T$-odd terms, in light of possible radiative corrections that could be induced from 
the nonphoton sectors in the standard-model extension. We have shown that the radiative corrections at one loop are finite, so it is unnecessary at this level of renormalization to include a $C P T$-odd term in the original theory. The finite corrections are gauge invariant but ambiguous, a situation somewhat reminiscent of the usual anomaly calculations. However, if the theory underlying the standard-model extension is anomaly free, the $C P T$-odd effects in the photon sector can be neglected at this level. Generalizations of this argument may apply at higher loops.

For the $C P T$-even sector, we have demonstrated by explicit one-loop calculation that divergent radiative corrections appear. A term of this type therefore must be present in the original theory. An experimental search for the associated renormalized coupling based, for example, on cosmological birefringence could be performed. It is remarkable that physics associated with the Planck scale might produce observable effects in measurements made at the largest scales in the universe.

\section{ACKNOWLEDGMENTS}

We thank R. Bluhm and P. Kronberg for discussions. This work was supported in part by the United States Department of Energy under Grant No. DE-FG02-91ER40661.
[1] V. A. Kostelecký and S. Samuel, Phys. Rev. Lett. 63, 224 (1989); 66, 1811 (1991); Phys. Rev. D 39, 683 (1989); 40, 1886 (1989); V. A. Kostelecký and R. Potting, Nucl. Phys. B359, 545 (1991); Phys. Lett. B 381, 89 (1996).

[2] D. Colladay and V. A. Kostelecký, Phys. Rev. D 55, 6760 (1997).

[3] V. A. Kostelecký and R. Potting, in Gamma Ray-Neutrino Cosmology and Planck Scale Physics, edited by D. B. Cline (World Scientific, Singapore, 1993), hep-th/9211116; Phys. Rev. D 51, 3923 (1995); D. Colladay and V. A. Kostelecký, Phys. Lett. B 344, 259 (1995); Phys. Rev. D 52, 6224 (1995); V. A. Kostelecký and R. Van Kooten, ibid. 54, 5585 (1996).

[4] B. Schwingenheuer et al., Phys. Rev. Lett. 74, 4376 (1995); L. K. Gibbons et al., Phys. Rev. D 55, 6625 (1997); R. Carosi et al., Phys. Lett. B 237, 303 (1990).

[5] OPAL Collaboration, R. Ackerstaff et al., Z. Phys. C 76, 401 (1997); DELPHI Collaboration, M. Feindt et al., DELPHI 97-98 CONF 80 (July 1997).

[6] V. A. Kostelecký, Phys. Rev. Lett. 80, 1818 (1998).

[7] P. B. Schwinberg, R. S. Van Dyck, Jr., and H. G. Dehmelt, Phys. Lett. A 81, 119 (1981); R. S. Van Dyck, Jr., P. B. Schwinberg, and H. G. Dehmelt, Phys. Rev. D 34, 722 (1986); L. S. Brown and G. Gabrielse, Rev. Mod. Phys. 58, 233 (1986); R. S. Van Dyck, Jr., P. B. Schwinberg, and H. G. Dehmelt, Phys. Rev. Lett. 59, 26 (1987); G. Gabrielse et al., ibid. 74, 3544 (1995).

[8] R. Bluhm, V. A. Kostelecký, and N. Russell, Phys. Rev. Lett. 79, 1432 (1997); Phys. Rev. D 57, 3932 (1998).

[9] O. Bertolami et al., Phys. Lett. B 395, 178 (1997).

[10] R. Bluhm, V. A. Kostelecký, and N. Russell, preprint IUHET 388 (1998).

[11] M. Charlton et al., Phys. Rep. 241, 65 (1994); Antihydrogen, edited by J. Eades (Baltzer, Geneva, 1993).

[12] V. A. Kostelecký and C. D. Lane (in preparation).

[13] V. W. Hughes, H. G. Robinson, and V. Beltran-Lopez, Phys. Rev. Lett. 4, 342 (1960); R. W. P. Drever, Philos. Mag. 6, 683 (1961); J. D. Prestage et al., Phys. Rev. Lett. 54, 2387 (1985); S. K. Lamoreaux et al., ibid. 57, 3125 (1986); T. E. Chupp et al., ibid. 63, 1541 (1989).

[14] P. A. M. Dirac, Proc. R. Soc. London B209, 291 (1951); W. Heisenberg, Rev. Mod. Phys. 29, 269 (1957); P. G. O. Freund,
Acta Phys. Austriaca 14, 445 (1961); J. D. Bjorken, Ann. Phys. (N.Y.) 24, 174 (1963); I. Bialynicki-Birula, Phys. Rev. 130, 465 (1963); G. S. Guralnik, ibid. 136, B1404 (1964); Y. Nambu, Prog. Theor. Phys. Suppl. Extra 190 (1968); T. Eguchi, Phys. Rev. D 14, 2755 (1976).

[15] H. B. Nielsen and I. Picek, Nucl. Phys. B211, 269 (1983).

[16] S. W. Hawking, Phys. Rev. D 14, 2460 (1976); Commun. Math. Phys. 87, 395 (1982).

[17] J. Ellis, J. L. Lopez, N. E. Mavromatos, and D. V. Nanopoulos, Phys. Rev. D 53, 3846 (1996).

[18] For a review, see, for example, C. M. Will, Theory and Experiment in Gravitational Physics (Cambridge University Press, Cambridge, England, 1993).

[19] Note that the definitions of these terms differ slightly from those in Ref. [2].

[20] See, for example, D. W. Hertzog et al., in Proceedings of the 1998 International Conference on Particles, Fields and Cosmology, edited by P. Nath (to be published).

[21] See, for example, Particle Data Group, R. M. Barnett et al., Phys. Rev. D 54, 1 (1996).

[22] S. M. Carroll, G. B. Field, and R. Jackiw, Phys. Rev. D 41, 1231 (1990).

[23] H. A. Lorentz, Verh. Kon. Akad. Wetensch. 18 (1878).

[24] See, for example, M. Born and E. Wolf, Principles of Optics, 5th ed. (Pergamon, New York, 1975); L. D. Landau, E. M. Lifshitz, and L. P. Pitaevskii, Electrodynamics of Continuous Media, 2nd ed. (Pergamon, New York, 1984).

[25] A. Sommerfeld, Ann. Phys. (Leipzig) 44, 177 (1914); L. Brioullin, ibid. 44, 203 (1914).

[26] See, for example, Handbook of Chemistry and Physics, edited by R. C. Weast, 56th ed. (CRC, Cleveland, Ohio, 1975).

[27] W.-T. Ni, Phys. Rev. Lett. 38, 301 (1977).

[28] The existing limit on the photon mass based on direct measurements is $m_{\gamma}<6 \times 10^{-25} \mathrm{GeV}$. See L. Davis, Jr., A. S. Goldhaber, and M. M. Nieto, Phys. Rev. Lett. 35, 1402 (1975); This bound, obtained from observation of the Jovian magnetosphere during the Pioneer-10 mission, might eventually be improved through measurements on the solar magnetosphere. See V. A. Kostelecký and M. M. Nieto, Phys. Lett. B 317, 223 (1993).

[29] P. Haves and R. G. Conway, Mon. Not. R. Astron. Soc. 173, 
53P (1975); J. N. Clarke, P. P. Kronberg, and M. SimardNormandin, ibid. 190, 205 (1980).

[30] B. Nodland and J. P. Ralston, Phys. Rev. Lett. 78, 3043 (1997); 79, 1958 (1997); astro-ph/9706126.

[31] S. M. Carroll and G. B. Field, Phys. Rev. Lett. 79, 2394 (1997); D. J. Eisenstein and E. F. Bunn, ibid. 79, 1957 (1997); J. P. Leahy, astro-ph/9704285; J. F. C. Wardle, R. A. Perley, and M. H. Cohen, Phys. Rev. Lett. 79, 1801 (1997); T. J. Loredo, E. E. Flanagan, and I. M. Wasserman, Phys. Rev. D 56, 7507 (1997).
[32] A. P. Lightman and D. L. Lee, Phys. Rev. D 8, 64 (1973).

[33] S. Coleman and S. Glashow, Phys. Lett. B 405, 249 (1997).

[34] See, for example, C. Q. Geng and R. E. Marshak, Comments Nucl. Part. Phys. 18, 331 (1989).

[35] L. Rosenberg, Phys. Rev. 129, 2786 (1963).

[36] S. L. Adler, Phys. Rev. 177, 2426 (1969); J. S. Bell and R. Jackiw, Nuovo Cimento A 60, 47 (1969).

[37] W. A. Bardeen, Phys. Rev. 84, 1848 (1969).

[38] S. L. Adler and W. A. Bardeen, Phys. Rev. 182, 1517 (1969). 